\title{
Gravity dual of Navier-Stokes equation in a rotating frame through parallel transport
}

\author{
Sumit Dey, ${ }^{1, *}$ Shounak De, ${ }^{2, \dagger}$ and Bibhas Ranjan Majhi $\circledast^{1, \hbar}$ \\ ${ }^{1}$ Department of Physics, Indian Institute of Technology Guwahati, Guwahati 781039, Assam, India \\ ${ }^{2}$ Department of Applied Mathematics and Theoretical Physics, University of Cambridge, \\ Wilberforce Road, Cambridge CB3 OWA, United kingdom
}

(Received 2 May 2020; accepted 10 August 2020; published 3 September 2020)

\begin{abstract}
The fluid-gravity correspondence documents a precise mathematical map between a class of dynamical spacetime solutions of the Einstein field equations of gravity and the dynamics of its corresponding dual fluid flows governed by the Navier-Stokes (NS) equations of hydrodynamics. This striking connection has been explored in several dynamics-based approaches and has surfaced in various forms over the past four decades. In a recent construction, it has been shown that the manifold properties of geometric duals are in fact intimately connected to the dynamics of incompressible fluids, thus bypassing the conventional on-shell standpoints. Following such a prescription, we construct the geometrical description that effectively captures the dynamics of an incompressible NS fluid with respect to a uniformly rotating frame. We propose the gravitational dual(s) described by bulk metric(s) in $(p+2)$ dimensions such that the equations of parallel transport of an appropriately defined bulk velocity vector field when projected onto an induced timelike hypersurface require that the incompressible NS equation of a fluid relative to a uniformly rotating frame be satisfied at the relevant perturbative order in $(p+1)$ dimensions. We argue that free fluid flows on manifold(s) described by the proposed metric(s) can be effectively considered as an equivalent theory of nonrelativistic viscous fluid dynamics with respect to a uniform rotating frame. We also present suggestive insights as to how spacetime rotation parameters encode information pertaining to the inertial effects in the corresponding fluid dual.
\end{abstract}

DOI: 10.1103/PhysRevD.102.064003

\section{INTRODUCTION}

The nonrelativistic incompressible Navier-Stokes (NS) equation $[1,2]$

$$
\dot{\vec{v}}+\vec{v} . \nabla \vec{v}+\vec{\nabla} P-\eta \nabla^{2} \vec{v}=0
$$

and the Einstein field equations of gravity

$$
G_{a b}=\frac{8 \pi G}{c^{4}} T_{a b}
$$

are two of the most important and well-studied differential equations in physics and mathematics. While the incompressible NS equation (1.1) universally governs the

\footnotetext{
*dey18@iitg.ac.in

†sd868@cam.ac.uk

bibhas.majhi@iitg.ac.in
}

Published by the American Physical Society under the terms of the Creative Commons Attribution 4.0 International license. Further distribution of this work must maintain attribution to the author(s) and the published article's title, journal citation, and DOI. Funded by SCOAP ${ }^{3}$. dynamics of fluids in the hydrodynamic limit, the Einstein equation (1.2) is known to universally govern the long-distance dynamics of gravitating systems. A precise mathematical bridge between Eqs. (1.1) and (1.2) and their solution spaces is well documented by the fluidgravity correspondence $[3,4]$. This striking connection relating the dynamics of gravity to those of fluid equations has gradually taken shape and surfaced in various forms over the past four decades.

One of the earliest works relating the dynamics of gravity and that of hydrodynamics appeared in the doctoral thesis of Damour [5], wherein there are suggestions of a connection between horizon and fluid dynamics. This work contains an expression now known as the Damour-NavierStokes (DNS) equation and it is known to govern the geometric data on any null surface. The same equation is also obtained in terms of coordinates adapted to a null surface [6,7] by projecting the Einstein equations of motion onto the null hypersurface (a similar analysis was also done in Ref. [8] for scalar-tensor gravity theory to obtain a DNSlike equation). Moreover, a corresponding action formulation of the same was given in detail in Ref. [9]. A connection in this regard was also obtained in the membrane paradigm approach by Price and Thorne in Ref. [10]. 
The membrane paradigm was applied in Ref. [11] in the context of asymptotically AdS spacetimes to show the dynamics of the membrane being described by the incompressible NS equation. In Ref. [12] the authors obtained an analogous DNS-type equation for both future outer trapped horizons and dynamical horizons (which are spacelike). One peculiarity of the DNS equation as obtained on a null horizon is that the bulk viscosity of the horizon fluid is negative. This makes the null horizon fluid unfit to have a connection with ordinary fluids. However, the authors of Ref. [12] showed that the horizon fluid on both future outer trapped horizons and the dynamical horizons have a positive value of the bulk viscosity. In the AdS/CFT context, it has been shown that the dissipative behavior of an anti-de Sitter (AdS) black hole agrees with the hydrodynamics of the holographically dual conformal field theory. In this approach the NS equation together with its corrections arise under a gradient expansion of the Einstein equations. This has been studied extensively and important works in this regard include Refs. [13-16]. More recently, in a cutoff surface approach by Bredberg et al. [17] it was shown by explicit construction that for every solution of the incompressible NS equation in $(p+1)$ dimensions there is a uniquely associated dual solution of the vacuum Einstein equations in $(p+2)$ dimensions. The metric of Ref. [17] was extended to all orders perturbatively via gradient expansion in Ref. [18], thus yielding higher-order corrections to the NS equation as well as the incompressibility condition. In Ref. [19] the authors generalized the cutoff surface approach by expounding on the dynamics of the dual field theory living on the boundary of AdS spacetime, provided the Dirichlet boundary conditions on the $r=r_{c}$ cutoff surface are ensured. The authors showed that there exists a critical radius as we go towards the horizon, beyond which a relativistic description of the fluid living on the cutoff surface is not valid because of the acausal propagation of sound modes. Allowing for nonrelativistic scaling, the authors retrieved the Ricci-flat gravitational duals to the incompressible NS equations. In Ref. [20] the authors provided a general approach to fluid/gravity correspondence, where the base metric is no longer the flat Rindler metric, but rather a generic static metric. The spacetime is endowed with a general bulk stress-energy tensor and an event horizon. This cutoff surface approach has been applied in various cases; see Refs. [21-23]. For example, it was extended for higher-curvature gravity theories [24-28] as well as for the AdS [29,30] and dS [31] gravity theories (for other theories, like black branes, see Ref. [32]). Very recently, two of the authors of this paper showed in Ref. [22] that an incompressible DNS-like equation can be obtained in the cutoff surface approach. In this case the obtained metric is a solution of the Einstein equations of motion in the presence of a particular type of matter. Also, a corresponding relativistic situation was discussed extensively in Ref. [33]. Symmetries of the vacuum Einstein equations were exploited to develop a formalism for solution-generating transformations of the corresponding NS fluid duals in Ref. [34]. The fluid description on the Kerr horizon was also explored extensively in Ref. [35] (see Ref. [36] for the isolated horizon case). The correspondence was also established for general rotating black holes yielding a Coriolis force term [37]. For extensive reviews of the fluid-gravity correspondence, see Refs. [3,4,38].

Having discussed the conventional approaches to this fascinating connection relating the dynamics of gravity to that of hydrodynamics, a novel interpretation of the same correspondence was established in a new setting. In a recent work by the authors of this paper [39], a new formalism was established to understand the fluid-gravity correspondence from a different standpoint. In the previous cutoff surface approach the underlying physics is that there exists a nontrivial map between the fluid side and the gravity side constrained by their dynamical equations of motion. This approach lays out the connection or duality between the dynamics of the incompressible fluid and that of the Einstein gravitational equations of motion via the conservation of the Brown-York stress tensor on the gravity side. However, fundamentally, the physics in Ref. [39] is quite different. Here the correspondence is between an incompressible fluid living in Minkowski spacetime and that of an appropriately defined bulk velocity field in curved spacetime. We then encode the dynamics of the bulk velocity field congruence in order to have a map between the fluid in the Minkowski spacetime and the bulk velocity field in the curved manifold. By dynamics, we impose that the acceleration of the congruence of the bulk velocity field on the $r=r_{c}$ timelike hypersurface is zero, i.e., the bulk velocity congruence is parallel transported on the $r=r_{c}$ slice. This allows us to have a map between the dynamics on both sides. The incompressible NS equation of the fluid is mapped to a "free" bulk velocity congruence on the $r=r_{c}$ hypersurface. The essence of the physics in Ref. [39] is that dynamics of the incompressible viscous fluid in Minkowski spacetime can be studied as the dynamics of a "free" parallel-transported bulk velocity field on the cutoff slice. As a result of the projection of the parallel transport being the analogue of the dynamics on the manifold side, all of the dynamical degrees of freedom of the fluid are encoded in the manifold properties of the spacetime. The constraint of the incompressibility condition on the fluid side is shown to naturally arise from the vanishing of the expansion parameter corresponding to the bulk velocity field. It is for this reason that the projection of the parallel transport equation of the bulk velocity field on the cutoff slice is so important in this framework. Moreover, this mapping between the two sides bypasses the Einstein field equations as an added advantage and hence is an off-shell duality between the incompressible fluid dynamics and parallel transport dynamics of a bulk velocity on the cutoff 
hypersurface. This approach to the fluid-gravity correspondence is completely different from existing works in this direction. This work [39] essentially forms the basis of the current paper, which attempts to construct a gravitational dual of the incompressible NS equation in a rotating frame, the details of which are discussed in the following paragraphs.

The notations used throughout the paper are clarified as follows. All lowercase latin letters denote the bulk spacetime coordinate indices and run from $a, b=0, \ldots, p+1$. The uppercase latin letters denote the transverse coordinates intrinsic to the hypersurface (i.e., the angular sector of the metric) and they run from $A, B=2, \ldots, p+1$ as the labels 0 and 1 have been chosen for time and radial coordinates, respectively.

\section{OUTLINE OF THE PAPER: A BRIEF REVIEW ON INCOMPRESSIBLE NS WITH RESPECT TO A ROTATING FRAME AND MOTIVATION}

The incompressible NS equation in an inertial frame of reference (1.1) takes the following form in index notation:

$$
\begin{aligned}
\partial_{\tau}^{\prime} v_{A}^{\prime}+v^{\prime C} \partial_{C}^{\prime} v_{A}^{\prime}+\partial_{A}^{\prime} P-\eta \partial^{\prime 2} v_{A}^{\prime} & =0, \\
\partial_{A}^{\prime} v^{\prime A} & =0,
\end{aligned}
$$

where $v_{A}^{\prime}$ denotes the fluid velocity with respect to an inertial coordinate system $\left(\tau^{\prime},\left\{x^{\prime A}\right\}\right)$. All of the derivatives are with respect to this primed inertial coordinate system. However, for a fluid relative to the rotating frame, inertial effects (like the Coriolis and centrifugal forces) need to be accounted for in the above equation when dealing with the dynamics of the fluid. The coordinates of the fluid element with respect to the rotating frame are designated as $\left(\tau,\left\{x^{A}\right\}\right)$. The transformations of the position vectors, the velocities, and the accelerations of the fluid element between the rotating frame and the inertial frame are given, respectively, via the relations

$$
\begin{aligned}
& \boldsymbol{x}=R \cdot \boldsymbol{x}^{\prime}, \\
& \boldsymbol{v}=\boldsymbol{R} \cdot \boldsymbol{v}^{\prime}-\boldsymbol{\Omega} \times \boldsymbol{x}, \\
& \boldsymbol{a}=\boldsymbol{R} \cdot \boldsymbol{a}^{\prime}-\boldsymbol{\Omega} \times(\boldsymbol{\Omega} \times \boldsymbol{x})-2(\boldsymbol{\Omega} \times \boldsymbol{v}),
\end{aligned}
$$

where $\boldsymbol{\Omega}$ is the uniform angular velocity of the rotating frame. $\boldsymbol{R}$ denotes the general time-dependent rotation matrix about any arbitrary plane. The centrifugal acceleration is given via $\boldsymbol{\Omega} \times(\boldsymbol{\Omega} \times \boldsymbol{x})$ and the Coriolis acceleration via $2(\boldsymbol{\Omega} \times \boldsymbol{v})$. Thus, the incompressible NS equation relative to a uniformly rotating frame for a nonrelativistic viscous fluid system (with no external forces) can be written as

$$
\begin{aligned}
\partial_{\tau} v_{A}+v^{B} \partial_{B} v_{A}+\partial_{A} P-\eta \partial^{2} v_{A}= & -2(\boldsymbol{\Omega} \times \boldsymbol{v})_{A} \\
& -(\boldsymbol{\Omega} \times(\boldsymbol{\Omega} \times \boldsymbol{x}))_{A}, \\
\partial_{A} v^{A}= & 0,
\end{aligned}
$$

where the Coriolis force is identified as $-2(\boldsymbol{\Omega} \times \boldsymbol{v})$ and the centrifugal force as $-\boldsymbol{\Omega} \times(\boldsymbol{\Omega} \times \boldsymbol{x})$.

The centrifugal force term can be identified as the gradient of a certain centrifugal potential (on the assumption that the origin of the rotating coordinate system lies on the axis of rotation) which can then be incorporated into the dynamical fluid pressure $P$ to identify an effective pressure term $P_{\text {eff }}$ [1]. The effective pressure $P_{\text {eff }}$ can then be identified as

$$
P_{\text {eff }}=P-\frac{1}{2}|\boldsymbol{\Omega} \times \boldsymbol{x}|^{2} .
$$

Thus, another form of the NS equation in the rotating frame is

$$
\begin{aligned}
\partial_{\tau} v_{A}+v^{C} \partial_{C} v^{A}+\partial_{A} P_{\text {eff }}-\eta \partial^{2} v_{A} & =-2(\boldsymbol{\Omega} \times \boldsymbol{v})_{A}, \\
\partial_{A} v^{A} & =0 .
\end{aligned}
$$

The details of this derivation can be found in Refs. [1,2].

In the above we found that the NS equation can be cast in two forms (2.3) and (2.5). Here, we attempt to construct the gravitational duals of both of them, in the manifoldbased approach to the fluid-gravity correspondence as established in Ref. [39]. Our main target will be to construct metrics that will lead to the NS equations when the parallel transport equation of a suitably chosen velocity vector is projected on the timelike hypersurface of these metrics. We first concentrate on finding the dual of Eq. (2.3), and then that for Eq. (2.5). We shall observe that, although the above two equations represent the same NS equation, the manifolds are distinctly different. However, the formalism of Ref. [39] yields equivalent NS equations (with respect to the rotating frame) for the fluid dual for both of these distinct metrics.

The basic organization of the paper is as follows. In Secs. IV and V, respectively, we begin by proposing two different bulk metrics in $(p+2)$ dimensions, on which we consider the equations of the projection of the parallel transport of an appropriately defined velocity vector field. We then show in Secs. IVA and VA that the projection of the parallel transport equations onto a timelike induced hypersurface requires that the fluid-dynamical NS equations (with respect to the rotating frame) be satisfied in $(p+1)$ dimensions. In Secs. IV B and V B we then show that the incompressibility condition of the fluid as viewed from the rotating frame derives from a vanishing expansion parameter $\theta$ when projected onto the same timelike induced hypersurface. We also identify the connections between the rotation parameter(s) on the gravity side and those in its 
fluid counterpart. In Sec. VI we provide further insight into the construction of the two proposed bulk metrics, which are genuinely curved backgrounds. By doing so, we show that the present considered proposed metrics are in no way related by a diffeomorphism of the metric presented in Ref. [39]. Finally, in Sec. VII we discuss the consequences of the two different gravity/metric duals we obtain for a NS fluid (relative to a rotating frame) in this parallel transport framework of the fluid-gravity correspondence and offer a few concluding remarks. The computation of the inverse(s) of the $(p+2)$-dimensional bulk metric(s), the relevant connection coefficients, and the order-wise calculations of the projected parallel transport equations and the expansion parameter $\theta$ are explicitly described in Appendixes A-F.

\section{SCALE INVARIANCE OF THE FLUID-DYNAMICAL EQUATIONS}

We propose two different metrics that effectively capture the dynamics of the viscous, NS fluid system relative to a uniformly rotating frame. Without using the Einstein field equations, we simply project the acceleration of an appropriately chosen bulk velocity congruence onto a specific chosen hypersurface for both of these metrics and demand that it be zero. Our main results-the specific forms of the manifolds-are obtained based on the scaling invariance of the NS equation. Therefore, we start this section with a discussion on this topic.

The incompressible NS equation (1.1) satisfies a wellknown scaling symmetry that is briefly stated as follows. If the solution space $\left(v^{A}, P\right)$ of the incompressible NS equation is scaled down by a certain hydrodynamic parameter $\epsilon$ as

$v_{A}^{\epsilon}\left(x^{A}, \tau\right)=\epsilon v_{A}\left(\epsilon x^{A}, \epsilon^{2} \tau\right) ; \quad P^{\epsilon}\left(x^{A}, \tau\right)=\epsilon^{2} P\left(\epsilon x^{A}, \epsilon^{2} \tau\right)$,

then the NS equation remains invariant under the above scaling transformations. A detailed derivation of the scale invariance of the NS equation can be found in Appendix A of Ref. [22]. The incompressible NS equation for a viscous fluid with respect to the rotating frame [Eq. (2.3) or, equivalently, Eq. (2.5)] also remains scale invariant if we identify that the uniform angular velocity $\boldsymbol{\Omega}$ scales as

$$
\Omega_{A}^{\epsilon}=\epsilon^{2} \Omega_{A} .
$$

The justification of this comes from the fact that angular velocity has dimensions of the inverse of time $\tau$. Since for the scale invariance $\tau$ scales as order $\mathcal{O}\left(\epsilon^{-2}\right)$, the components of the angular velocity scale as order $\mathcal{O}\left(\epsilon^{2}\right)$. Thus, via the hydrodynamic scaling $\epsilon$ we can generate a class of solutions parametrized by $\left(v_{A}^{\epsilon}, P^{\epsilon}\right)$. The hydrodynamic scalings of the dynamical variables - the constant angular velocity components along the spatial and temporal derivatives-follow as

$$
\begin{aligned}
& v_{A} \sim \mathcal{O}(\epsilon), \quad P \sim \mathcal{O}\left(\epsilon^{2}\right), \quad \Omega_{A} \sim \mathcal{O}\left(\epsilon^{2}\right), \\
& \partial_{A} \sim \mathcal{O}(\epsilon), \quad \partial_{\tau} \sim \mathcal{O}\left(\epsilon^{2}\right) .
\end{aligned}
$$

\section{FLUID DYNAMICS VIA PARALLEL TRANSPORT: CHOICE I}

For the proposed metric we follow the methodology applied in Ref. [17] and lay out the metric order by order in terms of the hydrodynamic scaling parameter $\epsilon$.

We propose a metric of the form

$$
\begin{aligned}
d s_{p+2}^{2}= & g_{a b} d x^{a} d x^{b}=-r d \tau^{2}+2 d \tau d r+d x_{A} d x^{A} \\
& -2 \beta_{A} f(r) d \tau d x^{A}-2 \beta_{A} g(r) d r d x^{A} \\
& +\left(a_{3}\left(x^{A} \Omega_{A} x^{B} \Omega_{B}\right)+a_{4}\left(\delta^{A B} \Omega_{A} \Omega_{B} \delta_{C D} x^{C} x^{D}\right)\right) d \tau^{2} \\
& -\left(\frac{2 a_{1}}{r_{c}} \partial_{A} P+2 a_{2} \partial^{2} v_{A}-\frac{4}{r_{c}} f(r) v^{D} \partial_{D} \beta_{A}\right) d x^{A} d r \\
& +\mathcal{O}\left(\epsilon^{4}\right) .
\end{aligned}
$$

The metric is written in such a way that the first line is $\mathcal{O}\left(\epsilon^{0}\right)$ and is simply the flat Rindler metric written in ingoing Eddington-Finkelstein coordinates, the second line is $\mathcal{O}\left(\epsilon^{1}\right)$, the third line is $\mathcal{O}\left(\epsilon^{2}\right)$, and the fourth line is $\mathcal{O}\left(\epsilon^{3}\right) . a_{1}, a_{2}$, $a_{3}$, and $a_{4}$ serve as constants whose values will be fixed later. We impose the condition that $\beta_{A}$ scales as order $\epsilon^{1}$. The present metric matches the proposed metric in Ref. [39] (in $a_{3}$ of Ref. [39] set to zero) if we set the constants $a_{3}$ and $a_{4}$ as well as $\beta_{A}$ equal to zero. The metric expanded to order $\mathcal{O}\left(\epsilon^{2}\right)$ serves as the base metric upon which the perturbation at order $\mathcal{O}\left(\epsilon^{3}\right)$ has been added. Initially, at this metric level, the set $\left\{\Omega_{A}\right\}$ are just some uniform components that we demand to scale as order $\mathcal{O}\left(\epsilon^{2}\right)$. No identification of $\left\{\Omega_{A}\right\}$ at this point can be made with the overall uniform angular velocity components for the fluid to be described. The same goes for the set $\left\{\beta_{A}\right\}$. Similarly, at the metric level, the fields $P\left(\tau, x^{A}\right)$ and $\left\{v_{A}\left(\tau, x^{B}\right)\right\}$ are not to be initially identified with the pressure perturbations and the velocity components of the fluid. All that we require at this metric level is that the fields $P\left(\tau, x^{A}\right)$ and $\left\{v_{A}\left(\tau, x^{B}\right)\right\}$ scale as order $\mathcal{O}\left(\epsilon^{2}\right)$ and $\mathcal{O}\left(\epsilon^{1}\right)$, respectively, in terms of the hydrodynamic scaling parameter $\epsilon$. The analogy/correspondence will emerge only after the formal machinery of the projection of the parallel transport equation has been applied.

We now explain the physical interpretation behind the construction of such a metric. We consider the effect of the following coordinate transformation on a fourdimensional Minkowski metric $d s^{2}=\eta_{a b} d x^{a} d x^{b}$ with $\eta:=$ $\operatorname{diag}\{-1,+1,+1,+1\}$ and $\left\{x^{a}\right\}:=\{t, x, y, z\}$ :

$$
\begin{aligned}
& \tilde{t}=t, \\
& \tilde{x}=x \cos (\omega t)+y \sin (\omega t), \\
& \tilde{y}=-x \sin (\omega t)+y \cos (\omega t), \\
& \tilde{z}=z .
\end{aligned}
$$


Under the effect of this coordinate transformation, where $\omega$ is a constant, the Minkowski metric becomes

$$
\begin{aligned}
d s^{2}= & -\left[1-\omega^{2}\left(\tilde{x}^{2}+\tilde{y}^{2}\right)\right] d \tilde{t}^{2}+2 \omega(\tilde{x} d \tilde{y}-\tilde{y} d \tilde{x}) d \tilde{t} \\
& +d \tilde{x}^{2}+d \tilde{y}^{2}+d \tilde{z}^{2} .
\end{aligned}
$$

It can be verified that in the nonrelativistic limit the geodesic equation of motion in this manifold reduces to the usual Newton's laws for a free particle in a rotating frame, incorporating both the Coriolis and centrifugal effects. The above discussion indicates that the coeffcients of $d \tau d x^{A}$ in our proposed metric are of the order $\mathcal{O}(\epsilon)$ whereas those for $d \tau^{2}$ are of order $\mathcal{O}\left(\epsilon^{2}\right)$. In our proposed metric, at this level $\beta_{A}$ is a function of $\left(\tau, x^{A}\right)$. However, $\Omega$ in our proposed metric is uniform and hence independent of $\tau, r$, and $\left\{x^{A}\right\}$. We recall that this is just a formal analogical way of proposing the present metric (4.1). A pertinent question might arise as to the emergence of the extra parameter $\beta_{A}$ in the proposed metric (4.1). It will be shown later after the formal machinery (of parallel transport) has been applied that $\left\{\Omega_{A}\right\}$ and $\left\{\beta_{A}\right\}$ must depend on each other for consistency.

We assume for the moment that in Eq. (4.1) $f(r)$ and $g(r)$ are smooth functions of only the radial coordinate, with the condition that $f\left(r=r_{c}\right) \neq 0$ and $g\left(r=r_{c}\right) \neq 0$. Here we also mention that $r=r_{c}$ is the location of the timelike hypersurface (in the bulk manifold) that we are interested in. The location is at any finite distance between the horizon $r=0$ and radial infinity. The base bulk metric here is

$$
\begin{aligned}
g_{a b}^{(0)} d x^{a} d x^{b}= & -r d \tau^{2}+2 d \tau d r+d x_{A} d x^{A}-2 \beta_{A} f(r) d \tau d x^{A} \\
& -2 \beta_{A} g(r) d r d x^{A}+\left(a_{3}\left(x^{A} \Omega_{A} x^{B} \Omega_{B}\right)\right. \\
& \left.+a_{4}\left(\delta^{A B} \Omega_{A} \Omega_{B} \delta_{C D} x^{C} x^{D}\right)\right) d \tau^{2}
\end{aligned}
$$

over which the perturbation

$$
\begin{aligned}
h_{a b}^{(3)} d x^{a} d x^{b}= & -\left(\frac{2 a_{1}}{r_{c}} \partial_{A} P+2 a_{2} \partial^{2} v_{A}\right. \\
& \left.-\frac{4}{r_{c}} f(r) v^{D} \partial_{D} \beta_{A}\right) d x^{A} d r
\end{aligned}
$$

to the order $\epsilon^{3}$ has been applied. We denote the perturbation as $h_{a b}^{(3)}$. As a result, the base bulk metric is curved, which can be checked by calculating the components of the Riemann curvature tensor $R_{b c d}^{a}$. The fields $P\left(\tau, x^{A}\right)$ and $v^{A}\left(\tau, x^{A}\right)$ are independent of the radial coordinate $r$. We shall show that the perturbation $h_{a b}^{(3)}$ in the proposed metric contains information about all of the forcing terms in the Navier-Stokes equation (relative to the rotating frame) for a viscous incompressible fluid. This proposed metric acts as the metric/gravity dual to the nonrelativistic fluid- dynamical equations written in a rotating coordinate system. Projecting the acceleration of the bulk fluid congruence in this given spacetime onto the timelike hypersurface $r=r_{c}$ and demanding that it be zero, we obtain the corresponding fluid-dynamical equation (and in the process lose general covariance).

\section{A. Parallel transport of the velocity field}

All of the kinematical and dynamical quantities of interest to us (which are defined for the entire spacetime manifold) will be projected onto the $r=r_{c}$ timelike hypersurface. The projection tensor onto the hypersurface $r=r_{c}$ is given by

$$
\gamma_{a b}=g_{a b}-n_{a} n_{b},
$$

where $n_{a}$ is the unit normal on this hypersurface. The hypersurface being timelike, its unit normal satisfies $n^{a} n_{a}=+1$ (spacelike unit normal). Since the base bulk metric is of the order $\mathcal{O}\left(\epsilon^{2}\right)$, the computation of $n_{a}$ (on the $r=r_{c}$ hypersurface) yields

$$
\begin{aligned}
\left.n_{\tau}\right|_{r=r_{c}}= & 0, \\
\left.n_{r}\right|_{r=r_{c}}= & \frac{1}{\sqrt{r_{c}}}+\frac{1}{r_{c}^{3 / 2}}\left\{a_{3}\left(x^{A} \Omega_{A} x^{B} \Omega_{B}\right)\right. \\
& \left.+a_{4}\left(\delta^{A B} \Omega_{A} \Omega_{B} \delta_{C D} x^{C} x^{D}\right)\right\} \\
& -\delta^{A B} \beta_{A} \beta_{B}\left(f^{2}+2 r f g+r^{2} g^{2}\right)+\mathcal{O}\left(\epsilon^{4}\right), \\
\left.\left\{n_{A}\right\}\right|_{r=r_{c}}= & 0 .
\end{aligned}
$$

The calculation for the components of the normal to the hypersurface has been carried up to $\mathcal{O}\left(\epsilon^{2}\right)$. The components of the projection tensor [to order $\mathcal{O}\left(\epsilon^{2}\right)$ ] on the $r=r_{c}$ slice follow as

$$
\begin{aligned}
\gamma_{\tau \tau}= & -r_{c}+a_{3}\left(x^{A} \Omega_{A} x^{B} \Omega_{B}\right)+a_{4}\left(\delta^{A B} \Omega_{A} \Omega_{B} \delta_{C D} x^{C} x^{D}\right) \\
\gamma_{\tau r}= & 1, \quad \gamma_{\tau A}=-\beta_{A} f\left(r_{c}\right), \\
\gamma_{r r}= & -\frac{1}{r_{c}}-\frac{1}{r_{c}^{2}}\left\{a_{3}\left(x^{A} \Omega_{A} x^{B} \Omega_{B}\right)+a_{4}\left(\delta^{A B} \Omega_{A} \Omega_{B} \delta_{C D} x^{C} x^{D}\right)\right. \\
& \left.-\delta^{A B} \beta_{A} \beta_{B}\left(f^{2}+2 r f g+r^{2} g^{2}\right)\right\} \\
\gamma_{r A}= & -\beta_{A} g\left(r_{c}\right), \quad \gamma_{A B}=\delta_{A B} .
\end{aligned}
$$

Raising these covariant projectors via the inverse metric tensor $g^{a b}$ (see Appendix A), we obtain the contravariant components of the projection tensor to the hypersurface $r=r_{c}$ up to the order $\mathcal{O}\left(\epsilon^{2}\right)$ as 


$$
\begin{aligned}
\gamma^{\tau \tau}= & -\frac{1}{r_{c}}-\frac{1}{r_{c}^{2}}\left\{a_{3}\left(x^{A} \Omega_{A} x^{B} \Omega_{B}\right)+a_{4}\left(\delta^{A B} \Omega_{A} \Omega_{B} \delta_{C D} x^{C} x^{D}\right)\right\} \\
& +\frac{f^{2}\left(r_{c}\right)}{r_{c}^{2}} \delta^{A B} \beta_{A} \beta_{B},
\end{aligned}
$$

$\gamma^{\tau r}=0, \quad \gamma^{\tau A}=-\frac{f\left(r_{c}\right)}{r_{c}} \delta^{A B} \beta_{B}$,

$\gamma^{r r}=0, \quad \gamma^{r A}=0, \quad \gamma^{A B}=\delta^{A B}+\frac{f^{2}\left(r_{c}\right)}{r_{c}} \delta^{A C} \delta^{B D} \beta_{C} \beta_{D}$

Now in the given spacetime manifold we define a bulk velocity field as $v^{a}=\left(1,0, v^{A}\right)$, such that there is no flow in the radial direction. The acceleration of the congruence of the velocity field is given via $a^{i}=v^{b} \nabla_{b} v^{i}$. We demand that the acceleration of this congruence as projected on the $r=r_{c}$ timelike hypersurface be zero. The component of the acceleration of the manifold fluid congruence on the $r=r_{c}$ slice is zero. This is represented by

$$
\left.\gamma_{a c} v^{b} \nabla_{b} v^{a}\right|_{r=r_{c}}=0
$$

It is in this sense that we are calling Eq. (4.10) a parallel transport equation or, more correctly, the projected parallel transport equation, i.e., the fluid congruence is "free" only on the $r=r_{c}$ hypersurface. Setting the free index $c$ to $\tau$, we obtain the lhs of Eq. (4.10) on the hypersurface $r=r_{c}$ of Eq. (4.1) as

$$
\begin{aligned}
\left.\gamma_{a \tau}\left(v^{b} \nabla_{b} v^{a}\right)\right|_{r=r_{c}}= & \gamma_{\tau \tau}\left(\Gamma_{\tau \tau}^{\tau}+2 \Gamma_{\tau A}^{\tau} v^{A}+\Gamma_{A B}^{\tau} v^{A} v^{B}\right)+\gamma_{r \tau}\left(\Gamma_{\tau \tau}^{r}+2 \Gamma_{\tau A}^{r} v^{A}+\Gamma_{A B}^{r} v^{A} v^{B}\right) \\
& \left.\gamma_{B \tau}\left(\partial_{\tau} v^{B}+v^{A} \partial_{A} v^{B}+\Gamma_{\tau \tau}^{B}+2 \Gamma_{\tau A}^{B} v^{A}+\Gamma_{A D}^{B} v^{A} v^{D}\right)\right|_{r=r_{c}} .
\end{aligned}
$$

The evaluation of Eq. (4.11) yields zero at orders $\mathcal{O}\left(\epsilon^{0}\right), \mathcal{O}\left(\epsilon^{1}\right), \mathcal{O}\left(\epsilon^{2}\right)$, and $\mathcal{O}\left(\epsilon^{3}\right)$, which is shown in detail in Appendix C. Hence, the projected parallel transport equation on the $r=r_{c}$ hypersurface with the free index $c=\tau$ is trivially satisfied up to order $\mathcal{O}\left(\epsilon^{3}\right)$.

Next, we turn our attention to the free index $c=r$ and have the following lhs of Eq. (4.10):

$$
\begin{aligned}
\left.\gamma_{a r}\left(v^{b} \nabla_{b} v^{a}\right)\right|_{r=r_{c}}= & \gamma_{\tau r}\left(\Gamma_{\tau \tau}^{\tau}+2 \Gamma_{\tau A}^{\tau} v^{A}+\Gamma_{A B}^{\tau} v^{A} v^{B}\right)+\gamma_{r r}\left(\Gamma_{\tau \tau}^{r}+2 \Gamma_{\tau A}^{r} v^{A}+\Gamma_{A B}^{r} v^{A} v^{B}\right) \\
& \left.\gamma_{A r}\left(\partial_{\tau} v^{A}+v^{B} \partial_{B} v^{A}+\Gamma_{\tau \tau}^{A}+2 \Gamma_{\tau B}^{A} v^{B}+\Gamma_{B C}^{A} v^{B} v^{C}\right)\right|_{r=r_{c}} .
\end{aligned}
$$

Evaluating Eq. (4.12) order by order, we see that it vanishes at $\mathcal{O}\left(\epsilon^{0}\right), \mathcal{O}\left(\epsilon^{1}\right)$, and $\mathcal{O}\left(\epsilon^{3}\right)$. However, at $\mathcal{O}\left(\epsilon^{2}\right)$, Eq. (4.12) yields a quantity proportional to $\frac{f^{2}\left(r_{c}\right)}{r_{c}}+2 f\left(r_{c}\right) g\left(r_{c}\right)+r_{c} g^{2}\left(r_{c}\right)$. The details are listed in Appendix C. The imposition of Eq. (4.10) implies

$$
f^{2}\left(r_{c}\right)+2 r_{c} f\left(r_{c}\right) g\left(r_{c}\right)+r_{c}^{2} g^{2}\left(r_{c}\right)=0,
$$

and as a consequence we obtain $g\left(r_{c}\right)=-\frac{f\left(r_{c}\right)}{r_{c}}$, which has to be satisfied on the $r=r_{c}$ timelike slice.

At this point we determine the covariant components of the velocity field. The contravariant components were defined as $v^{a}=\left(1,0, v^{A}\right)$. The covariant components of the velocity field (lowered via the base bulk metric) are

$$
\begin{aligned}
& v_{\tau}=-r-f \beta_{A} v^{A}+a_{3}\left(x^{A} \Omega_{A} x^{B} \Omega_{B}\right)+a_{4}\left(\delta^{A B} \Omega_{A} \Omega_{B} \delta_{C D} x^{C} x^{D}\right)+\mathcal{O}\left(\epsilon^{4}\right), \\
& v_{r}=1-g \beta_{A} v^{A}+\mathcal{O}\left(\epsilon^{4}\right), \quad v_{A}=-f \beta_{A}+\delta_{A B} v^{B} .
\end{aligned}
$$

We finally look at the projection of the parallel transport equation (4.10) with the free index $c=A$. As a consequence, for the lhs of Eq. (4.10) we obtain

$$
\begin{aligned}
\left.\gamma_{a A}\left(v^{b} \nabla_{b} v^{a}\right)\right|_{r=r_{c}}= & \gamma_{\tau A}\left(\Gamma_{\tau \tau}^{\tau}+2 \Gamma_{\tau D}^{\tau} v^{D}+\Gamma_{C D}^{\tau} v^{C} v^{D}\right)+\gamma_{r A}\left(\Gamma_{\tau \tau}^{r}+2 \Gamma_{\tau D}^{r} v^{D}+\Gamma_{C D}^{r} v^{C} v^{D}\right) \\
& \gamma_{B A}\left(\partial_{\tau} v^{B}+v^{C} \partial_{C} v^{B}+\Gamma_{\tau \tau}^{B}+2 \Gamma_{\tau D}^{B} v^{D}+\Gamma_{C D}^{B} v^{C} v^{D}\right) .
\end{aligned}
$$

Evaluating Eq. (4.15) order by order, we see that it vanishes at $\mathcal{O}\left(\epsilon^{0}\right), \mathcal{O}\left(\epsilon^{1}\right)$, and $\mathcal{O}\left(\epsilon^{2}\right)$. Evaluating the above equation on the $r=r_{c}$ cutoff hypersurface at $\mathcal{O}\left(\epsilon^{3}\right)$ yields (see Appendix C for details)

$$
\begin{aligned}
\mathcal{O}\left(\epsilon^{3}\right):\left.\gamma_{a A}\left(v^{b} \nabla_{b} v^{a}\right)\right|_{r=r_{c}}= & \partial_{\tau} v_{A}+v^{C} \partial_{C} v_{A}+f\left(r_{c}\right) v^{C} \partial_{C} \beta_{A}+\frac{r_{c}}{2}\left(\frac{a_{1}}{r_{c}} \partial_{A} P+a_{2} \partial^{2} v_{A}-\frac{2}{r_{c}} f\left(r_{c}\right) v^{D} \partial_{D} \beta_{A}\right) \\
& +f\left(r_{c}\right)\left(\partial_{A} \beta_{D}-\partial_{D} \beta_{A}\right) v^{D}-a_{3} \Omega_{A}\left(\Omega_{P} x^{P}\right)-a_{4}\left(\delta^{C D} \Omega_{C} \Omega_{D} \delta_{A P} x^{P}\right) .
\end{aligned}
$$


The validity of Eq. (4.10) with the free index $c=A$ at order $\mathcal{O}\left(\epsilon^{3}\right)$ imposes the requirement

$\partial_{\tau} v_{A}+v^{C} \partial_{C} v_{A}+\left(\frac{a_{1}}{2} \partial_{A} P+\frac{a_{2}}{2} r_{c} \partial^{2} v_{A}\right)+f\left(r_{c}\right)\left(\partial_{A} \beta_{D}-\partial_{D} \beta_{A}\right) v^{D}-a_{3} \Omega_{A}\left(\Omega_{P} x^{P}\right)-a_{4}\left(\delta^{C D} \Omega_{C} \Omega_{D} \delta_{A P} x^{P}\right)=0$.

Having finally arrived at Eq. (4.17), we want to make a correspondence between it and Eq. (2.3) (where $v$ denotes the velocity of the fluid element with respect to the rotating frame). It is at this level that we start making the necessary identifications between the quantities in the $(p+2)$-dimensional manifold sector and the respective quantities in the $(p+1)$-dimensional fluid dynamics sector. In order to make this parallel evident, the following identifications need to made. The fields $P\left(\tau, x^{A}\right)$ and $\left\{v_{A}\left(\tau, x^{B}\right)\right\}$ in the manifold sector [that scaled as order $\mathcal{O}\left(\epsilon^{2}\right)$ and $\mathcal{O}(\epsilon)$, respectively] are indeed the pressure perturbation and velocity field in the fluid sector. In the same way, we identify that the component $\Omega_{A}$ on the gravity side is equivalent to the uniform angular velocity of the frame in which the fluid is studied. We also identify that the uniform angular velocity components are related to the components $\left\{\beta_{A}\right\}$ via

$$
\left(\partial_{A} \beta_{B}-\partial_{B} \beta_{A}\right) v^{B}=-(\boldsymbol{\Omega} \times \boldsymbol{v})_{A},
$$

and as a result we can write

$$
\boldsymbol{\Omega}=\boldsymbol{\nabla} \times \boldsymbol{\beta}
$$

Hence, we see that at the metric level the components $\left\{\beta_{A}\right\}$ are not free parameters, but rather are constrained on $\left\{\Omega_{A}\right\}$. We can further constrain the functional form of $\beta$ via the fact that Eq. (2.3) represents the NS equation described in a uniformly rotating reference frame. Hence, we demand that the functional form of $\boldsymbol{\beta}$ be such that $\boldsymbol{\Omega}$ is a constant vector. One particular solution of Eq. (4.19) is

$$
\beta_{D}=\frac{1}{(p-1)} \epsilon_{B D}^{A} \Omega_{A} x^{B}+\phi_{D}(\tau)
$$

where $\phi(\tau)$ is any arbitrary function of $\tau$. We then constrain the functional form of $f(r)$ on the cutoff hypersurface as $f\left(r_{c}\right)=-2$, as a result of which $g\left(r_{c}\right)=\frac{2}{r_{c}}$. Identifying the constants $a_{i}$ in the proposed metric (4.1) as

$a_{1}=2, \quad a_{2}=-2, \quad a_{3}=-1, \quad a_{4}=1$

and the kinematic viscosity term $\eta$ as $\eta=r_{c}$, Eq. (4.17) becomes

$$
\begin{gathered}
\partial_{\tau} v_{A}+v^{C} \partial_{C} v_{A}+\partial_{A} P-\eta \partial^{2} v_{A}+2\left(\epsilon_{A B C} \Omega^{B} v^{C}\right) \\
+\Omega_{A}\left(\Omega_{P} x^{P}\right)-\left(\delta^{C D} \Omega_{C} \Omega_{D} \delta_{A P} x^{P}\right)=0 .
\end{gathered}
$$

It can be easily shown that the last two terms on the lhs of Eq. (4.22) are exactly the centrifugal force component $(\boldsymbol{\Omega} \times(\boldsymbol{\Omega} \times \boldsymbol{x}))_{A}$. So finally our formalism of the projection of the parallel transport equation on the timelike slice yields

$$
\begin{aligned}
& \partial_{\tau} v_{A}+v^{C} \partial_{C} v_{A}+\partial_{A} P-\eta \partial^{2} v_{A}+2(\boldsymbol{\Omega} \times \boldsymbol{v})_{A} \\
& +(\boldsymbol{\Omega} \times(\boldsymbol{\Omega} \times \boldsymbol{x}))_{A}=0
\end{aligned}
$$

which is identical to Eq. (2.3).

The above Eq. (4.23) is the Navier-Stokes equation (relative to the rotating frame) for a nonrelativistic, viscous fluid, with the last two terms being the inertial Coriolis and centrifugal forces, respectively, generated as a consequence of the relative fluid motion described in the rotating coordinate system. Thus, the inertial effects of the Coriolis and centrifugal forces are codified inside the proposed metric dual. In passing we mention that the centrifugal force can be expressed as the gradient of a certain centrifugal potential,

$$
(\boldsymbol{\Omega} \times(\boldsymbol{\Omega} \times \boldsymbol{x}))_{A}=-\partial_{A}\left(\frac{1}{2}|\boldsymbol{\Omega} \times \boldsymbol{x}|^{2}\right),
$$

in order to identify the effective pressure $P_{\text {eff }}$ as

$$
P_{\text {eff }}=P-\frac{1}{2}|\boldsymbol{\Omega} \times \boldsymbol{x}|^{2},
$$

thus obtaining Eq. (2.5) in the process.

\section{B. Incompressibility condition from the expansion scalar}

Now in order to quantify the incompressibility condition of the fluid on the dual metric side we look at the deviation tensor of the geodesic congruence of the bulk velocity field, and then project it onto the $r=r_{c}$ hypersurface. Following the analogy that the incompressibility implies density perturbations being zero over the continuum microscopic scales in the fluid side, we demand that the above relevant quantity must vanish. So we consider the tensor field $\nabla_{b} v_{a}$, which is the deviation of the geodesic fluid flow, and then we project this deviation tensor onto the $r=r_{c}$ timelike hypersurface. Basically, we evaluate the 
term $\Theta=\left.\gamma^{a b}\left(\nabla_{b} v_{a}\right)\right|_{r=r_{c}}$ which is the expansion scalar as seen on the timelike cutoff surface $r=r_{c}$. We shall see that the vanishing of this expansion scalar necessarily implies the incompressibility condition.

The corresponding projectors have been listed in Eq. (4.9). Hence, by expanding we have

$$
\begin{aligned}
\Theta= & -\gamma^{\tau \tau}\left(\Gamma_{\tau \tau}^{\tau} v_{\tau}+\Gamma_{\tau \tau}^{r} v_{r}+\Gamma_{\tau \tau}^{A} v_{A}\right) \\
& -2 \gamma^{\tau A}\left(\Gamma_{\tau A}^{\tau} v_{\tau}+\Gamma_{\tau A}^{r} v_{r}+\Gamma_{\tau A}^{A} v_{A}\right) \\
& +\gamma^{A B}\left(\partial_{A} v_{B}-\Gamma_{A B}^{\tau} v_{\tau}-\Gamma_{A B}^{r} v_{r}+\Gamma_{A B}^{D} v_{D}\right) .
\end{aligned}
$$

Evaluating the rhs of Eq. (4.26) reveals that it vanishes at orders $\mathcal{O}\left(\epsilon^{0}\right), \mathcal{O}\left(\epsilon^{1}\right)$, and $\mathcal{O}\left(\epsilon^{3}\right)$ (see Appendix $\mathrm{E}$ for details). The second-order term $\mathcal{O}\left(\epsilon^{2}\right)$ implies (see Appendix E)

$$
\mathcal{O}\left(\epsilon^{2}\right): \partial_{A} v^{A} .
$$

We impose the condition for the vanishing of the expansion scalar as evaluated on the $r=r_{c}$ cutoff hypersurface up to order $\mathcal{O}\left(\epsilon^{3}\right)$. This implies the incompressibility condition

$$
\partial_{A} v^{A}=0 .
$$

This is perhaps physically intuitive as the expansion scalar contains information as to the expansion or compression of the bulk geodesic velocity element, and its vanishing simply translates to the incompressibility condition on the fluid side.

\section{FLUID DYNAMICS VIA PARALLEL TRANSPORT: CHOICE II}

Again taking only the hydrodynamical scaling information from the fluid-dynamical side (3.3), we construct another metric expanded order by order in terms of the hydrodynamic scaling parameter $\epsilon$. We propose a metric of the form

$$
\begin{aligned}
d s_{p+2}^{2}= & g_{a b} d x^{a} d x^{b}=-r d \tau^{2}+2 d \tau d r+d x_{A} d x^{A} \\
& -2 \beta_{A} f(r) d \tau d x^{A}-2 \beta_{A} g(r) d r d x^{A} \\
& -\left(\frac{2 a_{1}}{r_{c}} \partial_{A} P_{\text {eff }}+2 a_{2} \partial^{2} v_{A}-\frac{4}{r_{c}} f(r) v^{D} \partial_{D} \beta_{A}\right) \\
& \times d x^{A} d r+\mathcal{O}\left(\epsilon^{4}\right) .
\end{aligned}
$$

The present metric again matches the proposed metric in Ref. [39] (in $a_{3}$ of Ref. [39] set to zero) provided $\beta_{A}$ has been set to zero. The first line is of order $\mathcal{O}\left(\epsilon^{0}\right)$ and is again the base Rindler metric, the second line is of order $\mathcal{O}\left(\epsilon^{1}\right)$, and the third line is of order $\mathcal{O}\left(\epsilon^{3}\right)$. Here we reiterate that $\beta_{A}\left(\tau, x^{B}\right)$ scales as order $\mathcal{O}\left(\epsilon^{1}\right)$ and at the metric level no concrete connection can be made between $\beta_{A}$ and the uniform angular velocity component $\Omega_{A}$ of the fluid side. However, intuitively, $\beta_{A}$ can be recognized along the lines of a "rotation" parameter of the spacetime. This has been done in analogy with the concept of frame dragging of inertial coordinates. If we assume for the moment that all of the metric coefficients are independent of the $\tau$ coordinate (i.e., the metric becomes stationary) and the $[A]$ th angular coordinate, then frame dragging becomes a generic feature in such stationary spacetimes where $g_{\tau[A]} \neq 0$. The square brackets on $[A]$ imply selecting only one angular coordinate $A$ out of the total $p$ coordinates. For such a metric there will be two conserved quantities: $p_{\tau}$ and $p_{[A]}$. The angular velocity of a particle dropped along the radial direction with zero conjugate momentum corresponding to the $[A]$ angular coordinate $\left(p_{[A]}=0\right)$ is $\frac{d x^{[A]}}{d \tau}=\frac{p^{[A]}}{p^{\tau}}=\omega(r, \bar{A}) \neq 0$, where $\bar{A}$ refers to all of the other angular coordinates without the single chosen $[A]$ coordinate. So a particle dropped radially will acquire a nonzero angular velocity. If we think passively about the particle being described in some local inertial frame where it is spatially at rest, then such inertial frames should be rotating with an angular velocity $\omega(r, \bar{A})$, and hence we say that inertial frames are dragged in this spacetime. It is in this sense that $\beta_{A}$, which in general is a function of $\left(\tau, x^{A}\right)$, is identified as a parameter that describes the rotation of the above-mentioned spacetime. Similar to metric choice $I$, the consistency conditions on $f\left(r_{c}\right)$ and $g\left(r_{c}\right)$ also hold for metric choice II. The same condition holds for the location of the timelike cutoff slice $r=r_{c}$. However, in Eq. (5.1) $P_{\text {eff }}\left(\tau, x^{A}\right)$ is a modification of the field $P\left(\tau, x^{A}\right)$ as presented in Eq. (4.1). However, we do demand that this modification be consistent with the scaling argument, i.e., $P_{\text {eff }}\left(\tau, x^{A}\right)$ scales as order $\mathcal{O}\left(\epsilon^{2}\right)$. At the end of the analysis we will decide what exact modification needs to be applied to $P_{\text {eff }}$ so that the duality between the manifold side and the fluid side is evident. The base bulk metric here is

$$
\begin{aligned}
g_{a b}^{(0)} d x^{a} d x^{b}= & -r d \tau^{2}+2 d \tau d r+d x_{A} d x^{A}-2 \beta_{A} f(r) d \tau d x^{A} \\
& -2 \beta_{A} g(r) d r d x^{A}
\end{aligned}
$$

over which the perturbation

$$
\begin{aligned}
h_{a b}^{(3)} d x^{a} d x^{b}= & -\left(\frac{2 a_{1}}{r_{c}} \partial_{A} P_{\text {eff }}+2 a_{2} \partial^{2} v_{A}\right. \\
& \left.-\frac{4}{r_{c}} f(r) v^{D} \partial_{D} \beta_{A}\right) d x^{A} d r
\end{aligned}
$$

to the order $\epsilon^{3}$ has been applied. We denote the perturbation as $h_{a b}^{(3)}$. As a result, the base bulk metric is curved, which can be checked by calculating the components of the Riemann curvature tensor $R_{b c d}^{a}$.

\section{A. Parallel transport of the velocity field}

The base part of the bulk metric proposal (5.1) (barring the perturbation at the third order) is written to order $\mathcal{O}(\epsilon)$. 
The unit normal on the $r=r_{c}$ timelike slice is evaluated to the first order in $\epsilon$. The computation yields

$$
n_{a}=\left(0, \frac{1}{\sqrt{r_{c}}}, \underset{\sim}{0}\right)
$$

The tilde underneath 0 implies that all of the angular components of the normal are zero. The components of the projection tensor [to order $\mathcal{O}(\epsilon)$ ] on the $r=r_{c}$ slice are

$$
\begin{aligned}
& \gamma_{\tau \tau}=-r_{c}, \quad \gamma_{\tau r}=1, \quad \gamma_{\tau A}=-\beta_{A} f\left(r_{c}\right), \\
& \gamma_{r r}=-\frac{1}{r_{c}}, \quad \gamma_{r A}=-\beta_{A} g\left(r_{c}\right), \quad \gamma_{A B}=\delta_{A B} .
\end{aligned}
$$

The contravariant components to order $\mathcal{O}(\epsilon)$ upon raising via the metric are

$$
\begin{array}{ll}
\gamma^{\tau \tau}=-\frac{1}{r_{c}}, \quad \gamma^{\tau r}=0, & \gamma^{\tau A}=-\frac{f\left(r_{c}\right)}{r_{c}} \delta^{A B} \beta_{B}, \\
\gamma^{r r}=0, & \gamma^{r A}=0, \quad \gamma^{A B}=\delta^{A B} .
\end{array}
$$

Again, for this proposed manifold defined via the metric (5.1) we define a bulk velocity field as $v^{a}=\left(1,0, v^{A}\right)$. We follow exactly the same algorithm as that for the metric choice $I$. The relevant information about the inverse metric components and the Christoffel symbols for the metric choice II are listed in Appendix B.

Setting the free index $c$ to $\tau$, for the metric (5.1) we evaluate the lhs of Eq. (4.10) order by order until $\mathcal{O}\left(\epsilon^{3}\right)$. We see that the lhs vanishes at $\mathcal{O}\left(\epsilon^{0}\right), \mathcal{O}\left(\epsilon^{1}\right), \mathcal{O}\left(\epsilon^{2}\right)$, and $\mathcal{O}\left(\epsilon^{3}\right)$, the details of which are shown in Appendix D. Hence, for the choice of the metric (5.1) the projection of the parallel transport equation on the $r=r_{c}$ hypersurface is trivially validated.

Next, we look at the lhs of Eq. (4.10) for the free index $c=r$. Evaluating, we find again that it vanishes at $\mathcal{O}\left(\epsilon^{0}\right)$, $\mathcal{O}\left(\epsilon^{1}\right)$, and $\mathcal{O}\left(\epsilon^{3}\right)$. However, at $\mathcal{O}\left(\epsilon^{2}\right)$ we have terms proportional to $\frac{f^{2}\left(r_{c}\right)}{r_{c}}+2 f\left(r_{c}\right) g\left(r_{c}\right)+r_{c} g^{2}\left(r_{c}\right)$. We are again presented with the same consistency condition on $f\left(r_{c}\right)$ and $g\left(r_{c}\right)$ for Eq. (4.10) to be valid, i.e., $g\left(r_{c}\right)=-\frac{f\left(r_{c}\right)}{r_{c}}$, which has to be satisfied on the $r=r_{c}$ timelike slice.

The covariant components of the velocity field (lowered via the base bulk metric) to $\mathcal{O}\left(\epsilon^{2}\right)$ are

$$
\begin{aligned}
& v_{\tau}=-r-f \beta_{A} v^{A}, \quad v_{r}=1-g \beta_{A} v^{A}, \\
& v_{A}=-f \beta_{A}+\delta_{A B} v^{B} .
\end{aligned}
$$

Evaluating the lhs of Eq. (4.10) with the free index $c=A$ reveals that it vanishes at $\mathcal{O}\left(\epsilon^{0}\right), \mathcal{O}\left(\epsilon^{1}\right)$, and $\mathcal{O}\left(\epsilon^{2}\right)$. At order $\mathcal{O}\left(\epsilon^{3}\right)$, we have

$$
\begin{aligned}
\mathcal{O}\left(\epsilon^{3}\right):\left.\gamma_{a A}\left(v^{b} \nabla_{b} v^{a}\right)\right|_{r=r_{c}}=\partial_{\tau} v_{A}+v^{C} \partial_{C} v_{A} \\
\quad+\frac{r_{c}}{2}\left(\frac{a_{1}}{r_{c}} \partial_{A} P_{\text {eff }}+a_{2} \partial^{2} v_{A}-\frac{2}{r_{c}} f\left(r_{c}\right) v^{D} \partial_{D} \beta_{A}\right) \\
\quad+f\left(r_{c}\right)\left(\partial_{A} \beta_{D}-\partial_{D} \beta_{A}\right) v^{D} .
\end{aligned}
$$

For Eq. (4.10) to be valid, we demand that

$$
\begin{gathered}
\partial_{\tau} v_{A}+v^{C} \partial_{C} v_{A}+\left(\frac{a_{1}}{2} \partial_{A} P_{\text {eff }}+\frac{a_{2}}{2} r_{c} \partial^{2} v_{A}\right) \\
+f\left(r_{c}\right)\left(\partial_{A} \beta_{D}-\partial_{D} \beta_{A}\right) v^{D}=0 .
\end{gathered}
$$

Having arrived at Eq. (5.9), we want to make a correspondence between it and Eq. (2.5). As before, we identify the field $v_{A}\left(\tau, x^{B}\right)$ written at the metric level as the velocity field of the fluid that needs to be described. On the same footing, we identify that the uniform angular velocity of the frame in which we study the fluid system is related to the "rotation parameter" $\left\{\beta_{A}\right\}$ on the gravity side via

$$
\left(\partial_{A} \beta_{B}-\partial_{B} \beta_{A}\right) v^{B}=-(\boldsymbol{\Omega} \times \boldsymbol{v})_{A},
$$

as a result of which we can establish the analogy

$$
\boldsymbol{\Omega}=\boldsymbol{\nabla} \times \boldsymbol{\beta} .
$$

We constrain the functional form of $f(r)$ on the cutoff hypersurface as $f\left(r_{c}\right)=-2$, as a result of which $g\left(r_{c}\right)=\frac{2}{r_{c}}$. Identifying the constants $a_{i}$ in the proposed metric (4.1) as

$$
a_{1}=2, \quad a_{2}=-2,
$$

and the kinematic viscosity term $\eta$ as $\eta=r_{c}$, Eq. (4.17) becomes

$\partial_{\tau} v_{A}+v^{C} \partial_{C} v_{A}+\partial_{A} P_{\text {eff }}-\eta \partial^{2} v+2\left(\epsilon_{A B C} \Omega^{B} v^{C}\right)=0$.

Thus, the correspondence with Eqs. (4.22) and (2.5) will be complete if we finalize the interpretation of $P_{\text {eff }}$. We demand that

$$
\begin{aligned}
P_{\text {eff }}= & P-\frac{1}{2}\left[x_{D} x^{D}\left(\partial^{A} \beta^{B}\right)\left(\partial_{A} \beta_{B}-\partial_{B} \beta_{A}\right)\right. \\
& \left.-x_{A} \epsilon^{A B C}\left(\partial_{B} \beta_{C}\right) x^{P} \epsilon_{P Q R}\left(\partial^{Q} \beta^{R}\right)\right] .
\end{aligned}
$$

All the raising and lowering of the components to the modifications (to $P$ ) are done via the Euclidean metric. The term added to $P$, i.e., $-\frac{1}{2}\left[x_{D} x^{D}\left(\partial^{A} \beta^{B}\right)\left(\partial_{A} \beta_{B}-\partial_{B} \beta_{A}\right)-\right.$ $\left.x_{A} \epsilon^{A B C}\left(\partial_{B} \beta_{C}\right) x^{P} \epsilon_{P Q R}\left(\partial^{Q} \beta^{R}\right)\right]$ can be identified as $-\frac{1}{2}(\boldsymbol{\Omega} \times \boldsymbol{x})^{2}$, where $\boldsymbol{x}$ denotes the transverse coordinates on the $r=r_{c}$ hypersurface, i.e., it is the position vector of the velocity element on the cutoff hypersurface and is in 
accordance with Eq. (2.4). The term $-\frac{1}{2}(\boldsymbol{\Omega} \times \boldsymbol{x})^{2}$ is identified as the centrifugal potential that needs to be added to the dynamical pressure to provide the effective pressure $P_{\text {eff }}$. We can clearly see here that $P_{\text {eff }}$ scales as order $\mathcal{O}\left(\epsilon^{2}\right)$.

The above Eq. (5.13) is the Navier-Stokes equation (with respect to the rotating frame) for a nonrelativistic viscous fluid, with the last term being the inertial Coriolis force, $2(\boldsymbol{v} \times \boldsymbol{\Omega})$, generated as a consequence of the relative fluid velocity described in the rotating coordinate system. Thus, the inertial effects of the Coriolis and centrifugal forces are codified in the proposed metric dual.

\section{B. Incompressibility condition from the expansion scalar}

We proceed as before and calculate the term $\Theta=$ $\left.\gamma^{a b}\left(\nabla_{b} v_{a}\right)\right|_{r=r_{c}}$, which is the expansion scalar as seen on the timelike cutoff surface $r=r_{c}$ for the manifold defined by Eq. (5.1). The corresponding projectors have been listed in Eq. (5.6). Hence, expanding, we have

$$
\begin{aligned}
\Theta= & \gamma^{a b}\left(\partial_{a} v_{b}+r \Gamma_{a b}^{\tau}+f \beta_{D} v^{D} \Gamma_{a b}^{\tau}-\Gamma_{a b}^{r}+g \beta_{D} v^{D} \Gamma_{a b}^{r}\right. \\
& \left.-\Gamma_{a b}^{D} v_{D}+f \beta_{D} \Gamma_{a b}^{D}\right)\left.\right|_{r=r_{c}} .
\end{aligned}
$$

Neglecting terms of order greater than $\mathcal{O}\left(\epsilon^{3}\right)$, the above Eq. (5.15) simplifies as

$$
\begin{aligned}
\Theta= & \frac{1}{r_{c}}\left(\Gamma_{\tau \tau}^{\tau} v_{\tau}+\Gamma_{\tau \tau}^{r} v_{r}+\Gamma_{\tau \tau}^{A} v_{A}\right) \\
& +\left(2 \frac{f\left(r_{c}\right)}{r_{c}} \delta^{A B} \beta_{B}\right)\left(\Gamma_{\tau A}^{\tau} v_{\tau}+\Gamma_{\tau A}^{r} v_{r}+\Gamma_{\tau A}^{D} v_{D}\right) \\
& +\delta^{A B}\left(\partial_{A} v_{B}-\Gamma_{A B}^{\tau} v_{\tau}-\Gamma_{A B}^{r} v_{r}+\Gamma_{A B}^{D} v_{D}\right) .
\end{aligned}
$$

Evaluating the rhs of Eq. (4.26) reveals that it vanishes at orders $\mathcal{O}\left(\epsilon^{0}\right), \mathcal{O}\left(\epsilon^{1}\right)$, and $\mathcal{O}\left(\epsilon^{3}\right)$ (see Appendix E for a derivation). The second-order term $\mathcal{O}\left(\epsilon^{2}\right)$ implies (see Appendix F)

$$
\mathcal{O}\left(\epsilon^{2}\right): \sim \partial_{A} v^{A} .
$$

We impose the condition for the vanishing of the expansion scalar as evaluated on the $r=r_{c}$ cutoff hypersurface up to order $\mathcal{O}\left(\epsilon^{3}\right)$. This implies the incompressibility condition

$$
\partial_{A} v^{A}=0 .
$$

\section{CONSTRUCTION OF THE PROPOSED METRICS: A DIFFERENT INTERPRETATION}

Now we will construct our two proposed metrics by a coordinate transformation on their respective base bulk metrics over which the perturbation at $\mathcal{O}\left(\epsilon^{3}\right)$ has been added to incorporate the forcing terms. About any event $\mathcal{P}$ in the manifold we employ the following coordinate transformations:

$$
\begin{aligned}
\tilde{x}^{A} & =x^{A}+\lambda \xi_{b c}^{A(3)} r, \\
\tilde{\tau} & =\tau, \\
\tilde{r} & =r,
\end{aligned}
$$

where $\xi_{b c}^{A(3)}$ is a 3 indexed component with $b$ and $c$ taking values of either $\tau$ or $r$. We impose that

$\xi_{b c}^{A(3)}=\delta^{A B}\left(\frac{a_{1}}{r_{c}} \partial_{B} P+a_{2} \partial^{2} v_{B}-\frac{2}{r_{c}} f(r) v^{D} \partial_{D} \beta_{B}\right)$

for the metric choice $I$ [Eq. (4.1)] and

$\xi_{b c}^{A(3)}=\delta^{A B}\left(\frac{a_{1}}{r_{c}} \partial_{B} P_{\text {eff }}+a_{2} \partial^{2} v_{B}-\frac{2}{r_{c}} f(r) v^{D} \partial_{D} \beta_{B}\right)$

for the metric choice $I I$ [Eq. (5.1)] such that the event $\mathcal{P}$ is taken to be the origin of both coordinate systems and where $\lambda$ is simply a constant that shall be fixed in due course. So applying these coordinate transformations on the initial base metric(s) (which are genuinely curved) does not change the overall structure of the spacetime. This is because the change in the Riemann curvature tensor $R^{a}{ }_{b c d}$ due to these coordinate transformations occurs at $\mathcal{O}\left(\epsilon^{4}\right)$. As a result, for the first metric we have

$$
\begin{aligned}
d \tilde{x}^{a}= & d x^{a} \\
& +\lambda\left[\delta^{A B}\left(\frac{a_{1}}{r_{c}} \partial_{B} P+a_{2} \partial^{2} v_{B}-\frac{2}{r_{c}} f(r) v^{D} \partial_{D} \beta_{B}\right)\right] d r \\
& +\mathcal{O}\left(\geq \epsilon^{4}\right) .
\end{aligned}
$$

Similarly, for the second metric we have

$$
\begin{aligned}
d \tilde{x}^{a}= & d x^{a} \\
& +\lambda\left[\delta^{A B}\left(\frac{a_{1}}{r_{c}} \partial_{B} P_{\text {eff }}+a_{2} \partial^{2} v_{B}-\frac{2}{r_{c}} f(r) v^{D} \partial_{D} \beta_{B}\right)\right] d r \\
& +\mathcal{O}\left(\geq \epsilon^{4}\right) .
\end{aligned}
$$

Imposing the above transformations (6.1) on the base metric written to the second order (4.4), we have 


$$
\begin{aligned}
d \tilde{s}_{p+2}^{2}= & -\tilde{r} d \tilde{\tau}^{2}+2 d \tilde{\tau} d \tilde{r}+d \tilde{x}_{A} d \tilde{x}^{A}-2 \beta_{A} f(\tilde{r}) d \tilde{\tau} d \tilde{x}^{A}-2 \beta_{A} g(\tilde{r}) d \tilde{r} d \tilde{x}^{A} \\
& +\left(a_{3}\left(\tilde{x}^{A} \Omega_{A} \tilde{x}^{B} \Omega_{B}\right)+a_{4}\left(\delta^{A B} \Omega_{A} \Omega_{B} \delta_{C D} \tilde{x}^{C} \tilde{x}^{D}\right)\right) d \tilde{\tau}^{2} \\
= & -r d \tau^{2}+2 d \tau d r+d x_{A} d x^{A}-2 \beta_{A} f(r) d \tau d x^{A}-2 \beta_{A} g(r) d r d x^{A} \\
& +\left(a_{3}\left(x^{A} \Omega_{A} x^{B} \Omega_{B}\right)+a_{4}\left(\delta^{A B} \Omega_{A} \Omega_{B} \delta_{C D} x^{C} x^{D}\right)\right) d \tau^{2} \\
& +\lambda\left(\frac{2 a_{1}}{r_{c}} \partial_{A} P+2 a_{2} \partial^{2} v_{A}-\frac{4}{r_{c}} f(r) v^{D} \partial_{D} \beta_{A}\right) d x^{A} d r+\mathcal{O}\left(\epsilon^{4}\right)
\end{aligned}
$$

Imposing the above transformations (6.1) on the base metric written to the first order (5.2), we have

$$
\begin{aligned}
d \tilde{s}_{p+2}^{2}= & -\tilde{r} d \tilde{\tau}^{2}+2 d \tilde{\tau} d \tilde{r}+d \tilde{x}_{A} d \tilde{x}^{A}-2 \beta_{A} f(\tilde{r}) d \tilde{\tau} d \tilde{x}^{A}-2 \beta_{A} g(\tilde{r}) d \tilde{r} d \tilde{x}^{A} \\
= & -r d \tau^{2}+2 d \tau d r+d x_{A} d x^{A}-2 \beta_{A} f(r) d \tau d x^{A}-2 \beta_{A} g(r) d r d x^{A} \\
& +\lambda\left(\frac{2 a_{1}}{r_{c}} \partial_{A} P_{\text {eff }}+2 a_{2} \partial^{2} v_{A}-\frac{4}{r_{c}} f(r) v^{D} \partial_{D} \beta_{A}\right) d x^{A} d r+\mathcal{O}\left(\epsilon^{4}\right)
\end{aligned}
$$

Setting the value of $\lambda=-1$, we obtain our proposed metrics up to $\mathcal{O}\left(\epsilon^{3}\right)$. So the effect of the forcing terms via the perturbation $h_{a b}^{(3)}$ at order $\mathcal{O}\left(\epsilon^{3}\right)$ present in the proposed metrics (4.1) and (5.1) can be thought of as the forces felt by observers moving in the spacetime defined by the base bulk metric, i.e., Eqs. (4.4) and (5.2), respectively, undergoing trajectories defined via Eq. (6.1). Hence, at least at the structural level, the original spacetimes (4.1) and (5.1) and the spacetimes (4.4) and (5.2), respectively, as observed by an observer following the trajectory (6.1) are inherently not different from each other at least to order $\mathcal{O}\left(\epsilon^{3}\right)$.

We mention that the base bulk metric (4.4), for the proposed metric (4.1), is a genuinely curved manifold as evident from the calculation of the curvature components. The nonzero components, up to order $\mathcal{O}\left(\epsilon^{1}\right)$, of $R^{a}{ }_{b c d}$ of the base metric up to order $\mathcal{O}\left(\epsilon^{1}\right)$ are evaluated to be

$$
\begin{gathered}
R_{r A r}^{r}=\frac{f^{\prime \prime} \beta_{A}}{2}, \quad R_{A \tau r}^{r}=-\frac{r f^{\prime \prime} \beta_{A}}{2}, \quad R_{\tau A r}^{r}=-\frac{r f^{\prime \prime} \beta_{A}}{2}, \\
R_{r \tau r}^{A}=\frac{\delta^{A B} \beta_{B} f^{\prime \prime}}{2}, \quad R_{A \tau r}^{\tau}=-\frac{f^{\prime \prime} \beta_{A}}{2}, \quad R_{\tau A r}^{\tau}=-\frac{f^{\prime \prime} \beta_{A}}{2} .
\end{gathered}
$$

This is also the case for the base metric (5.2). So, in general, even up to $\mathcal{O}\left(\epsilon^{1}\right)$, the Riemann curvature tensor has nonvanishing components. Therefore, both base bulk metrics (4.4) and (5.2), unlike that in Ref. [39], are curved. This is a very crucial difference between the earlier proposal and the present one. In this regard, it is worth mentioning that on the fluid side a simple set of coordinate transformations allows us to transform between the NS equation written in the inertial coordinates and the uniformly rotating noninertial coordinates. Therefore, one can expect that a similar argument can be applied to construct the metric for the rotating case. Hence it can be thought that a simple coordinate transformation on the proposed metric in Ref. [39] may provide the metric dual to the fluid seen from the rotating frame. In that respect, the base metric should be flat in both situations. But, unfortunately, this is not the case. As we mentioned above, the base metrics in the present discussion are curved in nature, so a coordinate transformation cannot connect them with the metric presented in Ref. [39]. This clearly shows that on the gravity side the idea is not so simple. Hence, the obtention of the gravity dual of the fluid equation in a rotating frame needs special attention. In addition, we found that the parameter that is connected to the intrinsic rotation of the spacetime (i.e., $\beta_{A}$ ) provides the rotational effect on the fluid side. This shows a clear correspondence between the parameters on both sides and, interestingly, the nonvanishing of $\beta_{A}$ guarantees the nonflatness of the base metrics. Hence, we feel that the analysis done here adds a nontrivial contribution to the subject of the fluid-gravity correspondence.

\section{DISCUSSIONS AND OUTLOOK}

We summarize our calculations as follows. We have proposed two bulk metrics in $(p+2)$ dimensions such that the base bulk metrics that act as the background are genuinely curved manifolds. To the zeroth order in the hydrodynamic parameter $\epsilon$, the background is essentially the flat Rindler spacetime for both spacetimes. To this background is added the perturbation $h_{a b}^{(3)}$ at $\mathcal{O}\left(\epsilon^{3}\right)$ which contains the information about both the pressure $P\left(\tau, x^{A}\right)$ and velocity $v_{A}\left(\tau, x^{B}\right)$ fields. The perturbation contains all of the "forcing" terms, i.e., the forces due to pressure gradients and the viscous effect. We then chose a bulk velocity vector field contained in this bulk spacetime defined as $v^{a}=\left(1,0, v^{A}\right)$. Our basic formalism involves 
the fact that this appropriately chosen bulk velocity has no component of its acceleration on the $r=r_{c}$ timelike hypersurface as evident from Eq. (4.10). This relation is what we call as the projection of the parallel transport equation. We looked at the projection of this parallel transport equation on a specific, safely chosen $r=r_{c}$ timelike hypersurface such that the location of the slice is away from the horizon (at $r=0$ ) and $r \rightarrow \infty$, as shown in Eq. (4.10). We demanded that the projected parallel transport equation onto the $r=r_{c}$ hypersurface is consistent to order $\mathcal{O}\left(\epsilon^{3}\right)$ [since the metric has been constructed to $\left.\mathcal{O}\left(\epsilon^{3}\right)\right]$. This implies that the incompressible NS equation (relative to the rotating frame) is valid at $\mathcal{O}\left(\epsilon^{3}\right)$ along with the consistency conditions on $f\left(r_{c}\right)$ and $g\left(r_{c}\right)$ being generated at $\mathcal{O}\left(\epsilon^{2}\right)$. The relevant centrifugal force is generated at order $\mathcal{O}\left(\epsilon^{3}\right)$ (via the parallel transport formalism) in the computation of the Christoffel connection component $\Gamma_{\tau \tau}^{A}$. Similarly, the Coriolis force is generated via the component $\Gamma_{\tau B}^{A}$. We then showed that demanding a vanishing expansion scalar for the bulk velocity field defined in the spacetime (as seen from the $r=r_{c}$ slice) leads to the incompressibility condition for the viscous fluid as observed from the rotating frame. Finally, we come up with a systematic method of constructing the proposed metrics (4.1) and (5.1) via a coordinate transformation on the base bulk metrics.

In our previous work [39], we proposed a metric dual to the incompressible nonrelativistic NS equation that contained (within the metric) all information about the forcing terms, i.e., the forces of the pressure gradient and the viscous effects. In that work, it was shown that the dynamics of a viscous, non-relativistic incompressible fluid in the Minkowski spacetime (described by the NS equation) is equivalent to the dynamics of a free fluid (described by the projected parallel transport equation) residing in a manifold with the proposed metric. The present work is an extension based on the formalism proposed in the earlier paper [39]. However, here the two proposed metrics are distinctly different, along with the fact that the base bulk metric(s) are no longer flat [Eqs. (4.4) and (5.2)]. Similarly, there are additional terms that occur in the perturbation at order $\mathcal{O}\left(\epsilon^{3}\right)$ for the two proposed metrics. The present two metrics carry all the information about the forcing terms along with the inertial centrifugal and Coriolis forces. This is because of the analogy that needs to be setup between the dynamics of an incompressible viscous fluid (as viewed from a rotating frame) with that of a free bulk fluid (being parallel transported along its own geodesic integral curves) residing on a manifold given by the proposed two metrics (4.1) and (5.1). When the fluid is to be described in a rotating coordinate system, then the metric is no longer diagonal in structure but rather involves cross terms between the temporal and spatial coordinates. On the dual metric side the effect of rotation is induced by the "rotation parameter" $\left\{\beta_{A}\right\}$ which causes the base bulk metric to no longer be flat. The projected parallel transport equation of the appropriately chosen bulk velocity field on the $r=r_{c}$ timelike slice generates the fluid dynamical equation which includes the Coriolis and centrifugal forces. The forcing terms of pressure and viscosity are encoded in $h_{a b}^{(3)}$ of the proposed metrics.

We can hence gain some new perspectives on the dynamical structure of the incompressible NS fluid equations relative to a rotating coordinate system. We can rewrite them in the $\boldsymbol{F}=m \boldsymbol{a}$ form,

$$
\begin{aligned}
\partial_{\tau} v_{A}+v^{D} \partial_{D} v_{A}= & -\partial_{A} P+\eta \partial^{2} v_{A}-(\boldsymbol{\Omega} \times(\boldsymbol{\Omega} \times \boldsymbol{x}))_{A} \\
& -2(\boldsymbol{\Omega} \times \boldsymbol{v})_{A},
\end{aligned}
$$

where the lhs is the total derivative for the velocity of the fluid element relative to the rotating system. The rhs contains the regular forcing terms due to pressure and viscosity along with the additional inertial centrifugal and Coriolis forces due to the system being described in a rotating frame. As is evident from Eq. (4.16), these forcing terms along with the inertial forces essentially arise from the evaluation of the relevant Christoffel symbols for the metrics (4.1) and (5.1). In our previous paper [39], all of the forcing terms were built inside the perturbation $h_{a b}^{(3)}$. In this paper, for the metric choice $I$ the pressure and viscous forces are generated from $h_{a b}^{(3)}$. The centrifugal force is generated through the metric component $g_{\tau \tau}^{(2)}$. The Coriolis force is generated from the rotation parameter $\beta\left(\tau, x^{A}\right)$ that shows its effect in the metric at order $\mathcal{O}(\epsilon)$. So if we were to "switch off" this perturbation of the fields by putting $P\left(\tau, x^{A}\right)=0$ and $v_{A}\left(\tau, x^{A}\right)=0$, then we would have a "forcing-free" fluid as described relative to a rotating coordinate system, i.e.,

$\partial_{\tau} v_{A}+v^{D} \partial_{D} v_{A}=(\boldsymbol{\Omega} \times(\boldsymbol{\Omega} \times \boldsymbol{x}))_{A}-2(\boldsymbol{\Omega} \times \boldsymbol{v})_{A}$.

Similarly, for the metric choice $I I$ the dynamical pressure (along with the centrifugal forces) and the viscous forces are incorporated in $h_{a b}^{(3)}$ of the metric. The Coriolis force is again generated from the rotation parameter $\beta\left(\tau, x^{A}\right)$ at order $\mathcal{O}(\epsilon)$ in the metric. Hence, the correspondence is that of a viscous incompressible fluid residing in a flat spacetime being dynamically equivalent to the geodesic flow of a free fluid (appropriately defined) in a curved manifold defined via Eq. (4.1) or Eq. (5.1). This actually in a sense parallels the interpretation where the dynamics of a particle interacting in a static gravitational field is locally indistinguishable from an equivalent accelerated frame, which has been expounded in Sec. 3.3 of Ref. [40].

Hence, we have observed that the two metrics in this paper account for all of the forcing as well as the inertial terms of the NS equation. Hence, the behavior of a free fluid in the proposed metrics can be considered as an 
equivalent theory of nonrelativistic viscous fluid dynamics relative to a uniformly rotating frame. As a result, a strong parallel can be drawn between this present analysis and the equivalence principle of gravity where an appropriate accelerated frame can locally mimic gravity. Thus, the duality presented here can be a dictionary between calculations on both the sides. Any calculation that may be hard to extract on the fluid side (of the incompressible viscous flow in the rotating frame) of the NS equation can be reflected by a calculation for a free fluid in the proposed metric spacetime (which incorporates both the effects of the forcing and the inertial effects), or vice versa. As a suggestive interpretation these metrics can be thought of as a complete geometrical description of the NS equation with respect to the rotating frame.

As a consequence of the projection of the parallel transport equation on the induced timelike hypersurface, we can obtain the NS equation. In fact, any corresponding alteration to the NS equation (relative to the rotating frame) at one's convenience can be supplemented with corresponding term(s) on the metric side, and the present algorithm would yield one's chosen equation. That is, one can add corrections to the NS equation and construct the dual metric easily. So the question naturally arises as to why we are fixated on the NS equation written in the rotating frame in our analysis. This is simply because we are within the purview of the nonrelativistic regime and the hydrodynamic $\operatorname{limit}(\epsilon \rightarrow 0)$. In this regime, the incompressible NS equation is universally the hydrodynamic limit to essentially any fluid system. Any corrections that can be thought of as coming from either kinetic theory the theory of strongly coupled fluids will necessarily get scaled away in this limit. We have hence constructed/formulated two metric duals to a viscous fluid system viewed from a uniformly rotating frame in this particular limit and singled out the NS equation as a consequence of parallel transport on these curved manifolds.

At this point we again stress that in the present analysis the Einstein equation has not been used to derive the correspondence between fluid dynamics and gravitational dynamics. In most of the earlier interpretations of fluid/ gravity correspondence [13-17,21-36] the Einstein equation played a pivotal role. The holographic approach to fluid/gravity correspondence, where the Einstein equation was interpreted as the NS equation on a timelike slice, is actually one of the possible ways to describe this correspondence. There is absolutely no requirement that the Einstein equation has to be used to connect the dynamics of both of these sectors. In our analysis we have shown this correspondence using the parallel transport equation as our guiding principle. Hence, our approach can be designated as an off-shell approach to fluid/gravity correspondence. The authors of Ref. [20] generalized the result of Ref. [17] by describing the dynamics of the fluid on the cutoff surface. They considered a general curved static metric rather than the flat Rindler metric, but kept the induced surface $r=r_{c}$ flat. By performing a set of scale transformations and Lorentz transformations, they obtained the seed metric for the relativistic fluid dual on the cutoff surface. However, our approach is based on the projection of the parallel transport equation on the $r=r_{c}$ surface. Moreover, in our case the $r=r_{c}$ surface is not flat because of the introduction of the rotation parameters $\beta_{A}$. Our work differs from their cutoff-surface-based approach again in the sense that ours is an off-shell analysis. However, we have not been able to consider the duality of the metric with a relativistic dual fluid.

We now mention some points that we perceive are the apparent benefits of such an off-shell approach to fluid/ gravity duality and thereby may provide possible future directions.

(1) The first point is the possibility of constructing an action for the fluid system from such a setup. The idea is as follows. The dynamics of the fluid are encoded in the manifold properties of the considered spacetime(s) which are a priori not required to be solutions of the Einstein equations. Using the duality between the fluid side and the manifold side via the projection of the parallel transport equations, the fluid system can be considered to be a collection of particles that are parallel transported on the $r=r_{c}$ hypersurface of the proposed spacetime(s). For such a fluid particle the action can be written as $\mathcal{A}=\int \sqrt{-g_{a b} u^{a} u^{b}} d \lambda$, where $u^{a}=d x^{a} / d \lambda$ is the velocity of the fluid particle and $g_{a b}$ is the metric of our proposed spacetimes. However, we do need to find a relation between $u^{a}$ and $v^{A}$, and then the action of the fluid particle can be expressed in terms of $v^{A}$. Extremizing such an action written for such a collection of fluid particles parallel transported on the $r=r_{c}$ slice in the proposed spacetimes might yield the required NS equation. In this way, an action principle of the NS equation may be constructed.

(2) As a classical correspondence, we have shown in our analysis the map between classical fluid-dynamical equations and the equations of the projected parallel transport of an appropriately defined bulk fluid velocity on the $r=r_{c}$ timelike slice of the given spacetime(s). However, there is a difficulty that arises when we try to have a quantum theory on both sides. In the on-shell approaches to fluid/gravity correspondence, we do have a quantum theory of the fluid. However, a quantum theory of gravity is as of yet in progress. Our off-shell approach may help to bypass this problem as we have not used the Einstein equations. On the fluid side, we have a many-body interacting theory of the fluid living in Minkowski spacetime. The analogous quantum theory on the manifold side in our approach is that of quantizing a 
collection of "free" or parallel-transported fluid particles in the background of the $r=r_{c}$ timelike slice of the proposed spacetime(s). This is basically semiclassical gravity where the background remains classical and we quantize matter fields in this background. Hence, the quantum theories on both sides can be related, bypassing the issues pertaining to quantum gravity.

(3) Another way in which this off-shell approach may help is in uncovering the microscopic degrees of freedom of the thermodynamic aspects of gravity. Our present formalism of fluid/gravity duality implies that all of the degrees of freedom (d.o.f.) of the fluid are possibly encoded in the manifold properties of the concerned proposed spacetime(s). Therefore, working knowledge of the microstructure of the fluid and hence its thermodynamics may help us to understand those of gravity. Moreover, it is now well known that one can associate quantities like entropy density and temperature with an arbitrary null hypersurface [41]. The null hypersurface does not need to be a solution to the Einstein equations. However, a proper origin of these quantities from the underlying microscopic structure of the spacetime is missing. Our off-shell approach to fluid/gravity duality may help to elucidate the microscopic d.o.f. of the gravity side since we have a mapping between the fluid and gravity sides. Previous approaches to fluid/gravity correspondence like the cutoff-surface method have explicitly used the Einstein field equations. Hence, we understand that in such approaches the d.o.f. of the fluid are encoded in the spacetimes that are required to be solutions to the Einstein equations. But as the thermodynamic entities can be assigned with an arbitrary null hypersurface and are related to the manifold properties which as such are not required to be solutions of the Einstein equations, it may be much more relevant to have an off-shell duality approach to identify the d.o.f. of the manifold. In this regard our present approach may shed some light on the thermodynamical origins of gravity.

In Ref. [39] we added a correction at order $\mathcal{O}\left(\epsilon^{3}\right)$ to the base flat Rindler metric that incorporated all of the forcing terms such that the demand of the projection of the parallel transport equation on the $r=r_{c}$ slice yielded the NS equation at $\mathcal{O}\left(\epsilon^{3}\right)$. Demanding that the projection of the expansion scalar on the $r=r_{c}$ slice vanish gave us the incompressibility condition at order $\mathcal{O}\left(\epsilon^{2}\right)$. It would definitely be interesting to construct the metric to all orders (as was done in Ref. [18]), such that we would also in our case retrieve the NS equation and the incompressibility condition along with the necessary corrections at higher orders. The basis of the construction of the metric in Ref. [18] to the $n$th order is as follows. Using the parallel of the hydrodynamic expansion of the fluid, the authors of Ref. [18] constructed the bulk expansion of the metric via a gradient expansion to all orders in $\epsilon$. Demanding Ricci flatness to all orders (which is a partial differential equation), the gradient expansion imposes a hierarchy between the derivatives which converts the partial differential equation into a series of coupled ordinary differential equations. Assuming that the metric has been written to order $\epsilon^{n-1}$, the authors added a new term $g_{a b}^{(n)}$ at order $\epsilon^{n}$, as a result of which the Ricci tensor at order $\epsilon^{n}$ is $R_{a b}^{(n)}=\delta R_{a b}^{(n)}+\hat{R}_{a b}^{(n)}$, where $\hat{R}_{a b}^{(n)}$ is the nonlinear contribution from the metric written until order $\epsilon^{n-1}$ and $\delta R_{a b}^{(n)}$ is the linearized contribution at order $\epsilon^{n}$ that contains only the $r$ derivatives. Demanding $R_{a b}^{(n)}=\delta R_{a b}^{(n)}+\hat{R}_{a b}^{(n)}=0$, the Ricci flatness condition is then integrated to find the corrections to the metric at order $\epsilon^{n}$ to the preexisting one written until order $\epsilon^{n-1}$. There are integrability conditions that need to be satisfied for these equations to be integrated. It turns out that the conservation of the Brown-York stress tensor on the $r=r_{c}$ slice at order $\epsilon^{n}$ ensures the validity of the integrability conditions. Mapping it onto the dual fluid side, this conservation yields the NS equation along with its corrections for all odd orders, while the conservation yields the incompressibility condition along with its corrections for all even orders.

Now we step back to see if it is possible in our present scheme to have a bulk construction of the metric to all orders in $\epsilon$, such that the projection of the parallel transport equation of an appropriately defined velocity field in this (bulk constructed to all orders) metric spacetime gives the NS equation along with its corrections. However, we have to build the metric with certain restrictions. Identifying these restrictions is nontrivial, and until now we have not been able to find them. Since we aim to present an off-shell description, it is not desirable to use any information from the Einstein equations. One such natural way of constructing the higher-order metric of Ref. [39] is via coordinate transformations. We have seen that the metric written to the third order in $\epsilon$ in Ref. [39] was generated by coordinate transformations on the base flat Rindler metric. Our goal is to construct the higher-order terms in the metric via such diffeomorphism transformations. We calculate $\delta g_{a b}^{(4)}$, which is the fourth-order contribution to the metric due to the application of the coordinate transformation $x^{a} \rightarrow x^{a}+$ $\xi^{(4) a}(x)$ on the $\epsilon^{3}$-order seed metric. Hence, one needs to find the diffeomorphism vector $\xi^{(4) a}(x)$ by taking the Lie variation of the metric i.e., $\delta g_{a b}^{(4)}=£_{\xi^{(4)}} g_{a b}$ constrained by certain conditions on the choice of the metric coeffcients. We shall state these conditions in a moment.. As a result, we have the following equations: 


$$
\begin{aligned}
& \delta g_{r r}^{(4)}=2 \partial_{r} \xi^{(4) \tau}, \\
& \delta g_{r \tau}^{(4)}=-r \partial_{r} \xi^{(4) \tau}+\partial_{r} \xi^{(4) r}, \\
& \delta g_{r A}^{(4)}=\partial_{r} \xi_{A}^{(4)}, \\
& \delta g_{\tau \tau}^{(4)}=-\xi^{(4) r}, \\
& \delta g_{\tau A}^{(4)}=0 \\
& \delta g_{A B}^{(4)}=0 .
\end{aligned}
$$

Because of the fact that we do not have boundary conditions prescribed for the components $\delta g_{r a}^{(4)}$ on the $r=r_{c}$ timelike slice (as for the $r=$ const surface $d r=0$ for any value of $g_{r a}$ ), we can set the constraint that $\delta g_{r a}^{(4)}=0$. In fact, this constraint needs to be extended to all orders such that $\delta g_{r a}^{(n)}=0$ and hence the $g_{r a}$ components are actually those of the metric components generated to the third order in $\epsilon$. That is, to all orders in the metric construction, we impose that

$$
\begin{aligned}
& g_{r r}=0, \quad g_{r \tau}=1, \\
& g_{r_{A}}=\left(\frac{a_{1}}{r_{c}} \partial_{A} P+a_{2} \partial^{2} v_{A}+\frac{a_{3}}{r_{c}} \partial_{A} v^{2}\right) .
\end{aligned}
$$

Now the above set of equations (7.3) need to be solved for $\xi^{(4) a}$ with the boundary conditions that $\delta g_{\tau \tau}^{(4)}, \delta g_{\tau A}^{(4)}$, and $\delta g_{A B}^{(4)}$ all vanish on the $r=r_{c}$ timelike slice. This fixes the integration constants upon the solution of Eq. (7.3). Now once we have solved for $\xi^{(4) a}$, we can apply the coordinate transformation $x^{a} \rightarrow x^{a}+\xi^{(4) a}(x)$ on the metric written to the third order in $\epsilon$ to generate the fourth-order correction to the metric. After this, we apply our machinery of the projection of the parallel transport equation of the bulk velocity field on the $r=r_{c}$ slice and generate the NS equation. The process can be iterated to construct the metric to the $n$th order in $\epsilon$ via coordinate transformations on the metric written to the $\epsilon^{n-1}$ order. The projected parallel transport equation of the bulk velocity field for the metric written to all orders in $\epsilon$ corresponds to the NS equation along with the higher order corrections.

Another possible way to construct the metric to higher orders may be via the metric compatibility condition i.e., $\nabla_{a} g_{b c}=0$. The idea is similar to that presented in Ref. [18]. We shall add a correction to the metric $\delta g_{a b}^{(n)}$ to the preconstructed metric written until order $\epsilon^{n-1}$. Since the condition is satisfied until our $\epsilon^{n-1}$-order metric, we choose the corrections at $\epsilon^{n}$ order such that the following is satisfied:

$$
\left(\nabla_{a} \widehat{g_{b c}}\right)^{(n)}+\nabla_{a}\left(\delta g_{b c}^{(n)}\right)=0
$$

where $\left(\widehat{\nabla_{a} g_{b c}}\right)^{(n)}$ is the $n$ th-order contribution due to the metric written until order $\epsilon^{n-1}$ and $\nabla_{a}\left(\delta g_{b c}^{(n)}\right)$ is the contribution due to $\delta g_{a b}^{(n)}$. This yields

$\partial_{r}\left(\delta g_{b c}^{(n)}\right)=\left(\nabla_{a} \hat{g_{b c}}\right)^{(n)}+\Gamma_{b a}^{i(0)}\left(\delta g_{i c}^{(n)}\right)+\Gamma_{c a}^{i(0)}\left(\delta g_{b i}^{(n)}\right)$,

which can be integrated to find the corrections to the metric. Next, with the imposition of the projection of the parallel transport equation of $v^{a}$ on the $r=r_{c}$ slice the higher-order corrections to the NS equation can be found. Then, from $\theta$, the corrections to the incompressibility condition can also be evaluated. A thorough investigation into this issue is required and we are certainly looking into it. Hence, nothing can be concretely stated right now. The work is in progress and will be reported in due time. In addition, we mention that this off-shell construction of the metric to all orders for the initial choice of the metrics (4.1) and (5.1) will be nontrivial since the base metrics in both cases are not Rindler flat, but rather genuinely curved spacetimes. As of now, we do not know which of the two procedures is correct, but we are looking into this. We certainly aim to report our investigations pertaining to these issues in the near future.

Finally, we mention that the above formalism can be extended to yield the Damour NS equation for a viscous fluid relative to a rotating frame if we modify the proposed metric by adding a certain term to $h_{a b}^{(3)}$ for both of the metrics. The term at order $\mathcal{O}\left(\epsilon^{3}\right)$ that does the job is $-\frac{2}{r_{c}} \partial_{A} v^{2}$. Following the exact same formalism outlined here, the rotating Damour NS equation has the form

$$
\begin{gathered}
\partial_{\tau} v_{A}+v^{C} \partial_{C} v_{A}+\frac{1}{2} \partial_{A} v^{2}+\partial_{A} P_{\mathrm{eff}}-\eta \partial^{2} v \\
+2\left(\epsilon_{A B C} \Omega^{B} v^{C}\right)=0 .
\end{gathered}
$$

Overall, we hope that the present discussion will shed more light on the subject of fluid-gravity correspondence as it provides a new method of investigation.

\section{APPENDIX A: THE INVERSE METRIC AND THE CHRISTOFFEL SYMBOLS OF METRIC CHOICE I}

We evaluate the inverse metric corresponding to Eq. (4.1) as a perturbation series over the flat metric $g_{a b}^{\text {(flat) }} d x^{a} d x^{b}=$ $-r d \tau^{2}+2 d \tau d r+d x_{A} d x^{A}$, with the perturbation identified as $H_{a b} d x^{a} d x^{b}=-2 \beta_{A} f(r) d \tau d x^{A}-2 \beta_{A} g(r) d r d x^{A}+$ $\left\{a_{3}\left(x^{A} \Omega_{A} x^{B} \Omega_{B}\right)+a_{4}\left(\delta^{A B} \Omega_{A} \Omega_{B} \delta_{C D} x^{C} x^{D}\right)\right\} d \tau^{2}-\left(\frac{2 a_{1}}{r_{c}} \partial_{A} P+\right.$ $\left.2 a_{2} \partial^{2} v_{A}-\frac{4}{r_{c}} f(r) v^{D} \partial_{D} \beta_{A}\right) d x^{A} d r$. The perturbation contains terms of order $\mathcal{O}(\epsilon), \mathcal{O}\left(\epsilon^{2}\right)$, and $\mathcal{O}\left(\epsilon^{3}\right)$. The inverse metric is written as a perturbation series over the flat Rindler base metric, 


$$
g^{a b}=g^{a b(0)}-H^{a b}+H^{a c} H_{c}^{b}-H^{a i} H_{i}^{k} H_{k}^{b}+\mathcal{O}\left(H^{4}\right),
$$

where all of the raising has been performed via the flat base metric. We list below the inverse metric up to order $\mathcal{O}\left(\epsilon^{3}\right)$ :

$$
\begin{gathered}
g^{\tau \tau}=g^{2} \delta^{A B} \beta_{A} \beta_{B}+\mathcal{O}\left(\epsilon^{4}\right), \\
g^{\tau r}=1+\left(r g^{2}+f g\right) \delta^{A B} \beta_{A} \beta_{B}+\mathcal{O}\left(\epsilon^{4}\right), \\
g^{\tau A}=g \delta^{A B} \beta_{B}+\delta^{A B}\left(\frac{a_{1}}{r_{c}} \partial_{B} P+a_{2} \partial^{2} v_{B}-\frac{2}{r_{c}} f(r) v^{D} \partial_{D} \beta_{B}\right)+\left(2 f g^{2}+r g^{3}\right) \delta^{A B} \delta^{C D} \beta_{B} \beta_{C} \beta_{D}+\mathcal{O}\left(\epsilon^{5}\right), \\
g^{r r}=r+\left(f^{2}+r^{2} g^{2}+2 r f g\right) \delta^{A B} \beta_{A} \beta_{B}-a_{3}\left(x^{A} \Omega_{A} x^{B} \Omega_{B}\right)-a_{4}\left(\delta^{A B} \Omega_{A} \Omega_{B} \delta_{C D} x^{C} x^{D}\right)+\mathcal{O}\left(\epsilon^{4}\right), \\
g^{r A}=(f+r g) \delta^{A B} \beta_{B}+r \delta^{A B}\left(\frac{a_{1}}{r_{c}} \partial_{B} P+a_{2} \partial^{2} v_{B}-\frac{2}{r_{c}} f(r) v^{D} \partial_{D} \beta_{B}\right)+\left(2 f^{2} g+3 r f g^{2}+r^{2} g^{3}\right) \delta^{A B} \delta^{C D} \beta_{B} \beta_{C} \beta_{D} \\
-g \delta^{A B} \beta_{B}\left[a_{3}\left(x^{P} \Omega_{P} x^{Q} \Omega_{Q}\right)+a_{4}\left(\delta^{C D} \Omega_{C} \Omega_{D} \delta_{P Q} x^{P} x^{Q}\right)\right]+\mathcal{O}\left(\epsilon^{5}\right), \\
g^{A B}=\delta^{A B}+\left(r g^{2}+2 f g\right) \delta^{A C} \delta^{B D} \beta_{C} \beta_{D}+\mathcal{O}\left(\epsilon^{4}\right) .
\end{gathered}
$$

In the same vein, we calculate the Christoffel symbols up to order $\mathcal{O}\left(\epsilon^{3}\right)$ :

$$
\begin{aligned}
& \Gamma_{\tau \tau}^{\tau}=\frac{1}{2}+\frac{1}{2}\left(r g^{2}+f g\right) \delta^{A B} \beta_{A} \beta_{B}+\mathcal{O}\left(\epsilon^{4}\right), \\
& \Gamma_{\tau r}^{\tau}=-\frac{1}{2}\left(g^{2}+f^{\prime} g\right) \delta^{A B} \beta_{A} \beta_{B}+\mathcal{O}\left(\epsilon^{4}\right), \\
& \Gamma_{\tau A}^{\tau}=\frac{1}{2} f^{\prime} \beta_{A}+\frac{1}{2}\left(r g^{2} f^{\prime}+f^{\prime} f g\right) \beta_{A} \delta^{C D} \beta_{C} \beta_{D}-\frac{1}{2} g \partial_{\tau} \beta_{A}+\frac{1}{2} f g \delta^{B C} \beta_{C}\left(\partial_{B} \beta_{A}-\partial_{A} \beta_{B}\right)+\mathcal{O}\left(\epsilon^{5}\right), \\
& \Gamma_{r r}^{\tau}=-g^{\prime} g \delta^{A B} \beta_{A} \beta_{B}+\mathcal{O}\left(\epsilon^{4}\right), \\
& \Gamma_{r A}^{\tau}=-\frac{1}{2} f^{\prime} g^{2} \beta_{A} \delta^{C D} \beta_{C} \beta_{D}+\frac{1}{2} g^{2} \delta^{B C} \beta_{C}\left(\partial_{B} \beta_{A}-\partial_{A} \beta_{B}\right)+\mathcal{O}\left(\epsilon^{5}\right), \\
& \Gamma_{A B}^{\tau}=-\frac{1}{2} g\left(\partial_{A} \beta_{B}+\partial_{B} \beta_{A}\right)+\mathcal{O}\left(\epsilon^{4}\right) \\
& \Gamma_{\tau \tau}^{r}=\frac{r}{2}+\frac{1}{2}\left(f^{2}+r^{2} g^{2}+2 r f g\right) \delta^{A B} \beta_{A} \beta_{B}-\frac{1}{2}\left[a_{3}\left(x^{A} \Omega_{A} x^{B} \Omega_{B}\right)+a_{4}\left(\delta^{A B} \Omega_{A} \Omega_{B} \delta_{C D} x^{C} x^{D}\right)\right]+\mathcal{O}\left(\epsilon^{4}\right), \\
& \Gamma_{\tau r}^{r}=-\frac{1}{2}-\frac{1}{2}\left(f^{\prime} f+r g f^{\prime}+r g^{2}+f g\right) \delta^{A B} \beta_{A} \beta_{B}+\mathcal{O}\left(\epsilon^{4}\right), \\
& \Gamma_{\tau A}^{r}=\frac{1}{2} r f^{\prime} \beta_{A}+\frac{1}{2}\left(f^{2} f^{\prime}+r^{2} g^{2} f^{\prime}+2 r f^{\prime} f g\right) \beta_{A} \delta^{B C} \beta_{B} \beta_{C}-\frac{r}{2} g \partial_{\tau} \beta_{A}+\frac{1}{2}\left(f^{2}+r f g\right) \delta^{B C} \beta_{C}\left(\partial_{B} \beta_{A}-\partial_{A} \beta_{B}\right) \\
& +a_{3} \Omega_{A}\left(\Omega_{D} x^{D}\right)+a_{4}\left(\delta^{P Q} \Omega_{P} \Omega_{Q} \delta_{A B} x^{B}\right)-\frac{1}{2} f^{\prime} \beta_{A}\left\{a_{3}\left(x^{C} \Omega_{C} x^{D} \Omega_{D}\right)+a_{4}\left(\delta^{C D} \Omega_{C} \Omega_{D} \delta_{P Q} x^{P} x^{Q}\right)\right\}+\mathcal{O}\left(\epsilon^{5}\right), \\
& \Gamma_{r r}^{r}=-\left(f g^{\prime}+r g g^{\prime}\right) \delta^{A B} \beta_{A} \beta_{B}+\mathcal{O}\left(\epsilon^{4}\right), \\
& \Gamma_{r A}^{r}=-\frac{1}{2} f^{\prime} \beta_{A}-\frac{1}{2}\left(f^{\prime} r g^{2}+f^{\prime} f g\right) \beta_{A} \delta^{B C} \beta_{B} \beta_{C}+\frac{1}{2} g \partial_{\tau} \beta_{A}+\frac{1}{2}\left(f g+r g^{2}\right) \delta^{B C} \beta_{C}\left(\partial_{B} \beta_{A}-\partial_{A} \beta_{B}\right)+\mathcal{O}\left(\epsilon^{5}\right), \\
& \Gamma_{A B}^{r}=-\frac{1}{2}(f+r g)\left(\partial_{A} \beta_{B}+\partial_{B} \beta_{A}\right)+\mathcal{O}\left(\epsilon^{4}\right),
\end{aligned}
$$




$$
\begin{aligned}
& \Gamma_{\tau \tau}^{A}=\frac{1}{2}(f+r g) \delta^{A B} \beta_{B}+\frac{r}{2} \delta^{A B}\left(\frac{a_{1}}{r_{c}} \partial_{B} P+a_{2} \partial^{2} v_{B}-\frac{2}{r_{c}} f(r) v^{D} \partial_{D} \beta_{B}\right) \\
& +\left(f^{2} g+\frac{3}{2} r f g^{2}+\frac{1}{2} r^{2} g^{3}\right) \delta^{A B} \delta^{C D} \beta_{B} \beta_{C} \beta_{D}-f \delta^{A B} \partial_{\tau} \beta_{B} \\
& -\frac{g}{2} \delta^{A B} \beta_{B}\left[a_{3}\left(x^{C} \Omega_{C} x^{D} \Omega_{D}\right)+a_{4}\left(\delta^{C D} \Omega_{C} \Omega_{D} \delta_{P Q} x^{P} x^{Q}\right)\right] \\
& -a_{3} \delta^{A B} \Omega_{B}\left(\Omega_{D} x^{D}\right)-a_{4}\left(\delta^{C D} \Omega_{C} \Omega_{D} x^{A}\right)+\mathcal{O}\left(\epsilon^{5}\right), \\
& \Gamma_{\tau r}^{A}=-\frac{1}{2}\left(f^{\prime}+g\right) \delta^{A B} \beta_{B}-\frac{1}{2} \delta^{A B}\left(\frac{a_{1}}{r_{c}} \partial_{B} P+a_{2} \partial^{2} v_{B}-\frac{2}{r_{c}} f(r) v^{D} \partial_{D} \beta_{B}\right) \\
& -\left(f g^{2}+\frac{1}{2} r g^{3}+\frac{1}{2} f^{\prime} r g^{2}+f^{\prime} f g\right) \delta^{A B} \delta^{C D} \beta_{B} \beta_{C} \beta_{D}-\frac{1}{2} g \delta^{A B} \partial_{\tau} \beta_{B}+\mathcal{O}\left(\epsilon^{5}\right), \\
& \Gamma_{\tau B}^{A}=\frac{1}{2}\left(f f^{\prime}+r g f^{\prime}\right) \delta^{A C} \beta_{B} \beta_{C}+\frac{f}{2} \delta^{A C}\left(\partial_{C} \beta_{B}-\partial_{B} \beta_{C}\right)+\mathcal{O}\left(\epsilon^{4}\right) \\
& \Gamma_{r r}^{A}=-g^{\prime} \delta^{A B} \beta_{B}-\left(r g^{2} g^{\prime}+2 f g g^{\prime}\right) \delta^{A B} \delta^{C D} \beta_{B} \beta_{C} \beta_{D}+\mathcal{O}\left(\epsilon^{5}\right) \\
& \Gamma_{r B}^{A}=-\frac{1}{2} f^{\prime} g \delta^{A C} \beta_{B} \beta_{C}+\frac{g}{2} \delta^{A C}\left(\partial_{C} \beta_{B}-\partial_{B} \beta_{C}\right)+\mathcal{O}\left(\epsilon^{4}\right), \\
& \Gamma_{B C}^{A}=-\left(f g+\frac{1}{2} r g^{2}\right) \delta^{A D} \beta_{D}\left(\partial_{B} \beta_{C}+\partial_{C} \beta_{B}\right)+\mathcal{O}\left(\epsilon^{5}\right)
\end{aligned}
$$

\section{APPENDIX B: THE INVERSE METRIC AND THE CHRISTOFFEL SYMBOLS OF METRIC CHOICE II}

In the same way, we evaluate the inverse metric corresponding to Eq. (5.1) as a perturbation series over the flat metric $g_{a b}^{(0)} d x^{a} d x^{b}=-r d \tau^{2}+2 d \tau d r+d x_{A} d x^{A}$, with the perturbation identified as $H_{a b} d x^{a} d x^{b}=-2 \beta_{A} f(r) d \tau d x^{A}-$ $2 \beta_{A} g(r) d r d x^{A}-\left(\frac{2 a_{1}}{r_{c}} \partial_{A} P_{\text {eff }}+2 a_{2} \partial^{2} v_{A}-\frac{4}{r_{c}} f(r) v^{D} \partial_{D} \beta_{A}\right) \times$ $d x^{A} d r$. The perturbation contains terms of order $\mathcal{O}\left(\epsilon^{1}\right)$ and
$\mathcal{O}\left(\epsilon^{3}\right)$. In fact, all of the calculations of the inverse metric components as well as the Christoffel connection components for metric choice $I I$ [Eq. (5.1)] can be retrieved from the calculations performed for metric choice $I$ [Eq. (4.1)] by setting $a_{3}=0, a_{4}=0$, and replacing $P\left(\tau, x^{A}\right)$ by $P_{\text {eff }}\left(\tau, x^{A}\right)$. Hence, we list out only the nontrivial changes, and the remaining components are identical to the ones computed in Appendix A. The changes to be made in the inverse metric components are

$$
\begin{gathered}
g^{\tau A}=g \delta^{A B} \beta_{B}+\delta^{A B}\left(\frac{a_{1}}{r_{c}} \partial_{B} P_{\text {eff }}+a_{2} \partial^{2} v_{B}-\frac{2}{r_{c}} f(r) v^{D} \partial_{D} \beta_{B}\right)+\left(2 f g^{2}+r g^{3}\right) \delta^{A B} \delta^{C D} \beta_{B} \beta_{C} \beta_{D}+\mathcal{O}\left(\epsilon^{5}\right) \\
g^{r r}=r+\left(f^{2}+r^{2} g^{2}+2 r f g\right) \delta^{A B} \beta_{A} \beta_{B}+\mathcal{O}\left(\epsilon^{4}\right) \\
g^{r A}=(f+r g) \delta^{A B} \beta_{B}+r \delta^{A B}\left(\frac{a_{1}}{r_{c}} \partial_{B} P_{\text {eff }}+a_{2} \partial^{2} v_{B}-\frac{2}{r_{c}} f(r) v^{D} \partial_{D} \beta_{B}\right) \\
+\left(2 f^{2} g+3 r f g^{2}+r^{2} g^{3}\right) \delta^{A B} \delta^{C D} \beta_{B} \beta_{C} \beta_{D}+\mathcal{O}\left(\epsilon^{5}\right)
\end{gathered}
$$

Similarly, the changes to be made in the Christoffel connection components are

$$
\Gamma_{\tau \tau}^{r}=\frac{r}{2}+\frac{1}{2}\left(f^{2}+r^{2} g^{2}+2 r f g\right) \delta^{A B} \beta_{A} \beta_{B}+\mathcal{O}\left(\epsilon^{4}\right),
$$




$$
\begin{gathered}
\Gamma_{\tau A}^{r}=\frac{1}{2} r f^{\prime} \beta_{A}+\frac{1}{2}\left(f^{2} f^{\prime}+r^{2} g^{2} f^{\prime}+2 r f^{\prime} f g\right) \beta_{A} \delta^{B C} \beta_{B} \beta_{C} \\
-\frac{r}{2} g \partial_{\tau} \beta_{A}+\frac{1}{2}\left(f^{2}+r f g\right) \delta^{B C} \beta_{C}\left(\partial_{B} \beta_{A}-\partial_{A} \beta_{B}\right)+\mathcal{O}\left(\epsilon^{5}\right), \\
\Gamma_{\tau \tau}^{A}=\frac{1}{2}(f+r g) \delta^{A B} \beta_{B}+\frac{r}{2} \delta^{A B}\left(\frac{a_{1}}{r_{c}} \partial_{B} P_{\mathrm{eff}}+a_{2} \partial^{2} v_{B}-\frac{2}{r_{c}} f(r) v^{D} \partial_{D} \beta_{B}\right) \\
+\left(f^{2} g+\frac{3}{2} r f g^{2}+\frac{1}{2} r^{2} g^{3}\right) \delta^{A B} \delta^{C D} \beta_{B} \beta_{C} \beta_{D}-f \delta^{A B} \partial_{\tau} \beta_{B}+\mathcal{O}\left(\epsilon^{5}\right), \\
\Gamma_{\tau r}^{A}=-\frac{1}{2}\left(f^{\prime}+g\right) \delta^{A B} \beta_{B}-\frac{1}{2} \delta^{A B}\left(\frac{a_{1}}{r_{c}} \partial_{B} P_{\mathrm{eff}}+a_{2} \partial^{2} v_{B}-\frac{2}{r_{c}} f(r) v^{D} \partial_{D} \beta_{B}\right) \\
-\left(f g^{2}+\frac{1}{2} r g^{3}+\frac{1}{2} f^{\prime} r g^{2}+f^{\prime} f g\right) \delta^{A B} \delta^{C D} \beta_{B} \beta_{C} \beta_{D}-\frac{1}{2} g \delta^{A B} \partial_{\tau} \beta_{B}+\mathcal{O}\left(\epsilon^{5}\right) .
\end{gathered}
$$

\section{APPENDIX C: PROJECTED PARALLEL TRANSPORT EQUATION (4.10) UP TO $\mathcal{O}\left(\epsilon^{3}\right)$ ORDER FOR METRIC CHOICE I}

We first consider the projected parallel transport equation with the free index $c=\tau$ [Eq. (4.11)],

$$
\begin{aligned}
\left.\gamma_{a \tau}\left(v^{b} \nabla_{b} v^{a}\right)\right|_{r=r_{c}}= & \gamma_{\tau \tau}\left(\Gamma_{\tau \tau}^{\tau}+2 \Gamma_{\tau A}^{\tau} v^{A}+\Gamma_{A B}^{\tau} v^{A} v^{B}\right)+\gamma_{r \tau}\left(\Gamma_{\tau \tau}^{r}+2 \Gamma_{\tau A}^{r} v^{A}+\Gamma_{A B}^{r} v^{A} v^{B}\right) \\
& \times \gamma_{B \tau}\left(\partial_{\tau} v^{B}+v^{A} \partial_{A} v^{B}+\Gamma_{\tau \tau}^{B}+2 \Gamma_{\tau A}^{B} v^{A}+\Gamma_{A D}^{B} v^{A} v^{D}\right) .
\end{aligned}
$$

At the following orders of the hydrodynamic expansion parameter, we have the rhs as

$$
\begin{gathered}
\mathcal{O}\left(\epsilon^{0}\right):-r_{c} \Gamma_{\tau \tau}^{\tau(0)}+\Gamma_{\tau \tau}^{r(0)}=0, \\
\mathcal{O}\left(\epsilon^{1}\right): \gamma_{\tau \tau}^{(0)}\left(\Gamma_{\tau \tau}^{\tau(1)}+2 \Gamma_{\tau A}^{\tau(0)} v^{A}\right)+\gamma_{r \tau}^{(0)}\left(\Gamma_{\tau \tau}^{r(1)}+\Gamma_{\tau A}^{r(0)} v^{A}\right)+\gamma_{B \tau}^{(1)}\left(\Gamma_{\tau \tau}^{B(0)}\right)=0, \\
\mathcal{O}\left(\epsilon^{2}\right): \gamma_{\tau \tau}^{(0)}\left(\Gamma_{\tau \tau}^{\tau(2)}+2 \Gamma_{\tau A}^{\tau(1)} v^{A}+\Gamma_{A B}^{\tau(0)} v^{A} v^{B}\right)+\gamma_{r \tau}^{(0)}\left(\Gamma_{\tau \tau}^{r(2)}+2 \Gamma_{\tau A}^{r(1)} v^{A}+\Gamma_{A B}^{r(0)} v^{A} v^{B}\right) \\
+\gamma_{B \tau}^{(1)}\left(\Gamma_{\tau \tau}^{B(1)}+2 \Gamma_{A \tau}^{B(0)} v^{A}\right)+\gamma_{\tau \tau}^{(2)}\left(\Gamma_{\tau \tau}^{\tau(0)}\right)=0, \\
\mathcal{O}\left(\epsilon^{3}\right): \gamma_{\tau \tau}^{(0)}\left(\Gamma_{\tau \tau}^{\tau(3)}+2 \Gamma_{\tau A}^{\tau(2)} v^{A}+\Gamma_{A B}^{\tau(1)} v^{A} v^{B}\right)+\gamma_{r \tau}^{(0)}\left(\Gamma_{\tau \tau}^{r(3)}+2 \Gamma_{\tau A}^{r(2)} v^{A}+\Gamma_{A B}^{r(1)} v^{A} v^{B}\right) \\
+\gamma_{B \tau}^{(1)}\left(\Gamma_{\tau \tau}^{B(2)}+2 \Gamma_{A \tau}^{B(1)} v^{A}+\Gamma_{A D}^{B(0)} v^{A} v^{D}\right)+\gamma_{\tau \tau}^{(2)}\left(\Gamma_{\tau \tau}^{\tau(1)}+\Gamma_{\tau A}^{\tau(0)} v^{A}\right)=0 .
\end{gathered}
$$

Next, we consider the projected parallel transport equation with the free index $c=r$ [Eq. (4.12)],

$$
\begin{aligned}
\left.\gamma_{a r}\left(v^{b} \nabla_{b} v^{a}\right)\right|_{r=r_{c}}= & \gamma_{\tau r}\left(\Gamma_{\tau \tau}^{\tau}+2 \Gamma_{\tau A}^{\tau} v^{A}+\Gamma_{A B}^{\tau} v^{A} v^{B}\right)+\gamma_{r r}\left(\Gamma_{\tau \tau}^{r}+2 \Gamma_{\tau A}^{r} v^{A}+\Gamma_{A B}^{r} v^{A} v^{B}\right) \\
& \times \gamma_{A r}\left(\partial_{\tau} v^{A}+v^{B} \partial_{B} v^{A}+\Gamma_{\tau \tau}^{A}+2 \Gamma_{\tau B}^{A} v^{B}+\Gamma_{B C}^{A} v^{B} v^{C}\right) .
\end{aligned}
$$

Expanding them order by order in terms of the hydrodynamic scaling parameter $\epsilon$, we obtain the rhs as

$$
\begin{gathered}
\mathcal{O}\left(\epsilon^{0}\right): \Gamma_{\tau \tau}^{\tau(0)}-\frac{1}{r_{c}}\left(\Gamma_{\tau \tau}^{r(0)}\right)=0, \\
\mathcal{O}\left(\epsilon^{1}\right): \gamma_{\tau r}^{(0)}\left(\Gamma_{\tau \tau}^{\tau(1)}+2 \Gamma_{\tau A}^{\tau(0)} v^{A}\right)+\gamma_{r r}^{(0)}\left(\Gamma_{\tau \tau}^{r(1)}+2 \Gamma_{\tau A}^{r(0)} v^{A}\right)+\gamma_{\mathrm{Br}}^{(1)}\left(\Gamma_{\tau \tau}^{B(0)}\right)=0,
\end{gathered}
$$




$$
\begin{aligned}
\mathcal{O}\left(\epsilon^{2}\right): & \gamma_{\tau r}^{(0)}\left(\Gamma_{\tau \tau}^{\tau(2)}+2 \Gamma_{\tau A}^{\tau(1)} v^{A}+\Gamma_{A B}^{\tau(0)} v^{A} v^{B}\right)+\gamma_{r r}^{(0)}\left(\Gamma_{\tau \tau}^{r(2)}+2 \Gamma_{\tau A}^{r(1)} v^{A}+\Gamma_{A B}^{r(0)} v^{A} v^{B}\right) \\
& +\gamma_{\mathrm{Br}}^{(1)}\left(\Gamma_{\tau \tau}^{B(1)}+2 \Gamma_{A \tau}^{B(0)} v^{A}\right)+\gamma_{r r}^{(2)}\left(\Gamma_{\tau \tau}^{r(0)}\right) \\
= & -\frac{1}{2 r_{c}} \delta^{C D} \beta_{C} \beta_{D}\left(f^{2}\left(r_{c}\right)+2 r_{c} f\left(r_{c}\right) g\left(r_{c}\right)+r_{c}^{2} g^{2}\right) \\
\mathcal{O}\left(\epsilon^{3}\right): & \gamma_{\tau r}^{(0)}\left(\Gamma_{\tau \tau}^{\tau(3)}+2 \Gamma_{\tau A}^{\tau(2)} v^{A}+\Gamma_{A B}^{\tau(1)} v^{A} v^{B}\right)+\gamma_{r r}^{(0)}\left(\Gamma_{\tau \tau}^{r(3)}+2 \Gamma_{\tau A}^{r(2)} v^{A}+\Gamma_{A B}^{r(1)} v^{A} v^{B}\right) \\
& +\gamma_{\mathrm{Br}}^{(1)}\left(\Gamma_{\tau \tau}^{B(2)}+2 \Gamma_{A \tau}^{B(1)} v^{A}+\Gamma_{A D}^{B(0)} v^{A} v^{D}\right)+\gamma_{r r}^{(2)}\left(\Gamma_{\tau \tau}^{r(1)}+2 \Gamma_{\tau A}^{r(0)} v^{A}\right)=0 .
\end{aligned}
$$

In a similar fashion, we consider the projection of the parallel transport equation on the $r=r_{c}$ cutoff hypersurface with the free index $c=A$, i.e., Eq. (4.15),

$$
\begin{aligned}
\left.\gamma_{a A}\left(v^{b} \nabla_{b} v^{a}\right)\right|_{r=r_{c}}= & \gamma_{\tau A}\left(\Gamma_{\tau \tau}^{\tau}+2 \Gamma_{\tau D}^{\tau} v^{D}+\Gamma_{C D}^{\tau} v^{C} v^{D}\right)+\gamma_{r A}\left(\Gamma_{\tau \tau}^{r}+2 \Gamma_{\tau D}^{r} v^{D}+\Gamma_{C D}^{r} v^{C} v^{D}\right) \\
& \times \gamma_{B A}\left(\partial_{\tau} v^{B}+v^{C} \partial_{C} v^{B}+\Gamma_{\tau \tau}^{B}+2 \Gamma_{\tau D}^{B} v^{D}+\Gamma_{C D}^{B} v^{C} v^{D}\right) .
\end{aligned}
$$

Expanding the above equation order by order in terms of $\epsilon$, we have the corresponding set

$$
\begin{gathered}
\mathcal{O}\left(\epsilon^{0}\right): \delta_{A B} \Gamma_{\tau \tau}^{B}=0, \\
\mathcal{O}\left(\epsilon^{1}\right):-f\left(r_{c}\right) \beta_{A} \Gamma_{\tau \tau}^{\tau(0)}-g\left(r_{c}\right) \beta_{A} \Gamma_{\tau \tau}^{r(0)}+\delta_{A B}\left(\Gamma_{\tau \tau}^{B(1)}+2 \Gamma_{\tau D}^{B(0)} v^{D}\right)=0, \\
\mathcal{O}\left(\epsilon^{2}\right): \gamma_{\tau A}^{(1)}\left(\Gamma_{\tau \tau}^{\tau(1)}+2 \Gamma_{\tau D}^{\tau(0)} v^{D}\right)+\gamma_{r A}^{(1)}\left(\Gamma_{\tau \tau}^{r(1)}+2 \Gamma_{\tau D}^{r(0)} v^{D}\right) \\
+\delta_{A B}\left(\Gamma_{\tau \tau}^{B(2)}+2 \Gamma_{\tau D}^{B(1)} v^{D}+\Gamma_{C D}^{B(0)} v^{C} v^{D}\right)=0, \\
\mathcal{O}\left(\epsilon^{3}\right): \gamma_{\tau A}^{(1)}\left(\Gamma_{\tau \tau}^{\tau(2)}+2 \Gamma_{\tau D}^{\tau(1)} v^{D}+\Gamma_{C D}^{\tau(0)} v^{C} v^{D}\right)+\gamma_{r A}^{(1)}\left(\Gamma_{\tau \tau}^{r(2)}+2 \Gamma_{\tau D}^{r(1)} v^{D}+\Gamma_{C D}^{r(0)} v^{C} v^{D}\right) \\
+\delta_{A B}\left(\partial_{\tau} v^{B}+v^{C} \partial_{C} v^{B}+\Gamma_{\tau \tau}^{B(3)}+2 \Gamma_{\tau D}^{B(2)} v^{D}+\Gamma_{C D}^{B(1)} v^{C} v^{D}\right)=\partial_{\tau} v_{A}+v^{C} \partial_{C} v_{A}+f\left(r_{c}\right) v^{C} \partial_{C} \beta_{A} \\
+\frac{r_{c}}{2}\left(\frac{a_{1}}{r_{c}} \partial_{A} P+a_{2} \partial^{2} v_{A}-\frac{2}{r_{c}} f\left(r_{c}\right) v^{D} \partial_{D} \beta_{A}\right)+f\left(r_{c}\right)\left(\partial_{A} \beta_{D}-\partial_{D} \beta_{A}\right) v^{D}-a_{3} \Omega_{A}\left(\Omega_{P} x^{P}\right) \\
-a_{4}\left(\delta^{C D} \Omega_{C} \Omega_{D} \delta_{A P} x^{P}\right) .
\end{gathered}
$$

\section{APPENDIX D: PROJECTED PARALLEL TRANSPORT EQUATION (4.10) UP TO $\mathcal{O}\left(\epsilon^{3}\right)$ ORDER FOR METRIC CHOICE II}

We first consider the projected autoparallel equation with the free index $c=\tau$ [Eq. (4.11)],

$$
\begin{aligned}
\left.\gamma_{a \tau}\left(v^{b} \nabla_{b} v^{a}\right)\right|_{r=r_{c}}= & \gamma_{\tau \tau}\left(\Gamma_{\tau \tau}^{\tau}+2 \Gamma_{\tau A}^{\tau} v^{A}+\Gamma_{A B}^{\tau} v^{A} v^{B}\right)+\gamma_{r \tau}\left(\Gamma_{\tau \tau}^{r}+2 \Gamma_{\tau A}^{r} v^{A}+\Gamma_{A B}^{r} v^{A} v^{B}\right) \\
& \times \gamma_{B \tau}\left(\partial_{\tau} v^{B}+v^{A} \partial_{A} v^{B}+\Gamma_{\tau \tau}^{B}+2 \Gamma_{\tau A}^{B} v^{A}+\Gamma_{A D}^{B} v^{A} v^{D}\right) .
\end{aligned}
$$

At the following orders of the hydrodynamic expansion parameter, we have the rhs as

$$
\begin{gathered}
\mathcal{O}\left(\epsilon^{0}\right):-r_{c} \Gamma_{\tau \tau}^{\tau(0)}+\Gamma_{\tau \tau}^{r(0)}=0, \\
\mathcal{O}\left(\epsilon^{1}\right):-r_{c}\left(\Gamma_{\tau \tau}^{\tau(1)}+2 \Gamma_{\tau A}^{\tau(0)} v^{A}\right)+\left(\Gamma_{\tau \tau}^{r(1)}+\Gamma_{\tau A}^{r(0)} v^{A}\right)-\left(f\left(r_{c}\right) \beta_{B}\right)\left(\Gamma_{\tau \tau}^{B(0)}\right)=0, \\
\mathcal{O}\left(\epsilon^{2}\right)-r_{c}\left(\Gamma_{\tau \tau}^{\tau(2)}+2 \Gamma_{\tau A}^{\tau(1)} v^{A}+\Gamma_{A B}^{\tau(0)} v^{A} v^{B}\right)+\left(\Gamma_{\tau \tau}^{r(2)}+2 \Gamma_{\tau A}^{r(1)} v^{A}+\Gamma_{A B}^{r(0)} v^{A} v^{B}\right) \\
-f\left(r_{c}\right) \beta_{B}\left(\Gamma_{\tau \tau}^{B(1)}+2 \Gamma_{A \tau}^{B(0)} v^{A}\right)=0,
\end{gathered}
$$




$$
\begin{aligned}
\mathcal{O}\left(\epsilon^{3}\right): & -r_{c}\left(\Gamma_{\tau \tau}^{\tau(3)}+2 \Gamma_{\tau A}^{\tau(2)} v^{A}+\Gamma_{A B}^{\tau(1)} v^{A} v^{B}\right)+\left(\Gamma_{\tau \tau}^{r(3)}+2 \Gamma_{\tau A}^{r(2)} v^{A}+\Gamma_{A B}^{r(1)} v^{A} v^{B}\right) \\
& -f\left(r_{c}\right) \beta_{B}\left(\Gamma_{\tau \tau}^{B(2)}+2 \Gamma_{A \tau}^{B(1)} v^{A}+\Gamma_{A D}^{B(0)} v^{A} v^{D}\right)=0 .
\end{aligned}
$$

Next, we consider the projected parallel transport equation with the free index $c=r$ [Eq. (4.12)],

$$
\begin{aligned}
\left.\gamma_{a r}\left(v^{b} \nabla_{b} v^{a}\right)\right|_{r=r_{c}}= & \gamma_{\tau r}\left(\Gamma_{\tau \tau}^{\tau}+2 \Gamma_{\tau A}^{\tau} v^{A}+\Gamma_{A B}^{\tau} v^{A} v^{B}\right)+\gamma_{r r}\left(\Gamma_{\tau \tau}^{r}+2 \Gamma_{\tau A}^{r} v^{A}+\Gamma_{A B}^{r} v^{A} v^{B}\right) \\
& \times \gamma_{A r}\left(\partial_{\tau} v^{A}+v^{B} \partial_{B} v^{A}+\Gamma_{\tau \tau}^{A}+2 \Gamma_{\tau B}^{A} v^{B}+\Gamma_{B C}^{A} v^{B} v^{C}\right) .
\end{aligned}
$$

Expanding them order by order in terms of the hydrodynamics scaling parameter $\epsilon$, we obtain the rhs as

$$
\begin{gathered}
\mathcal{O}\left(\epsilon^{0}\right): \Gamma_{\tau \tau}^{\tau(0)}-\frac{1}{r_{c}}\left(\Gamma_{\tau \tau}^{r(0)}\right)=0, \\
\mathcal{O}\left(\epsilon^{1}\right):-\left(\Gamma_{\tau \tau}^{\tau(1)}+2 \Gamma_{\tau A}^{\tau(0)} v^{A}\right)-\frac{1}{r_{c}}\left(\Gamma_{\tau \tau}^{r(1)}+2 \Gamma_{\tau A}^{r(0)} v^{A}\right)-\left(g\left(r_{c}\right) \beta_{B}\right)\left(\Gamma_{\tau \tau}^{B(0)}\right)=0, \\
\mathcal{O}\left(\epsilon^{2}\right):\left(\Gamma_{\tau \tau}^{\tau(2)}+2 \Gamma_{\tau A}^{\tau(1)} v^{A}+\Gamma_{A B}^{\tau(0)} v^{A} v^{B}\right)-\frac{1}{r_{c}}\left(\Gamma_{\tau \tau}^{r(2)}+2 \Gamma_{\tau A}^{r(1)} v^{A}+\Gamma_{A B}^{r(0)} v^{A} v^{B}\right) \\
-g\left(r_{c}\right) \beta_{B}\left(\Gamma_{\tau \tau}^{B(1)}+2 \Gamma_{A \tau}^{B(0)} v^{A}\right)=-\frac{1}{2 r_{c}} \delta^{C D} \beta_{C} \beta_{D}\left(f^{2}\left(r_{c}\right)+2 r_{c} f\left(r_{c}\right) g\left(r_{c}\right)+r_{c}^{2} g^{2}\right), \\
\mathcal{O}\left(\epsilon^{3}\right):\left(\Gamma_{\tau \tau}^{\tau(3)}+2 \Gamma_{\tau A}^{\tau(2)} v^{A}+\Gamma_{A B}^{\tau(1)} v^{A} v^{B}\right)-\frac{1}{r_{c}}\left(\Gamma_{\tau \tau}^{r(3)}+2 \Gamma_{\tau A}^{r(2)} v^{A}+\Gamma_{A B}^{r(1)} v^{A} v^{B}\right) \\
-g\left(r_{c}\right) \beta_{B}\left(\Gamma_{\tau \tau}^{B(2)}+2 \Gamma_{A \tau}^{B(1)} v^{A}+\Gamma_{A D}^{B(0)} v^{A} v^{D}\right)=0 .
\end{gathered}
$$

In a similar fashion, we consider the projection of the parallel transport equation on the $r=r_{c}$ cutoff hypersurface with the free index $c=A$, i.e., [Eq. (4.15)],

$$
\begin{aligned}
\left.\gamma_{a A}\left(v^{b} \nabla_{b} v^{a}\right)\right|_{r=r_{c}}= & \gamma_{\tau A}\left(\Gamma_{\tau \tau}^{\tau}+2 \Gamma_{\tau D}^{\tau} v^{D}+\Gamma_{C D}^{\tau} v^{C} v^{D}\right)+\gamma_{r A}\left(\Gamma_{\tau \tau}^{r}+2 \Gamma_{\tau D}^{r} v^{D}+\Gamma_{C D}^{r} v^{C} v^{D}\right) \\
& \times \gamma_{B A}\left(\partial_{\tau} v^{B}+v^{C} \partial_{C} v^{B}+\Gamma_{\tau \tau}^{B}+2 \Gamma_{\tau D}^{B} v^{D}+\Gamma_{C D}^{B} v^{C} v^{D}\right) .
\end{aligned}
$$

Expanding the above equation order by order in terms of $\epsilon$, we have the corresponding set

$$
\begin{gathered}
\mathcal{O}\left(\epsilon^{0}\right): \delta_{A B} \Gamma_{\tau \tau}^{B}=0 \\
\mathcal{O}\left(\epsilon^{1}\right):-f\left(r_{c}\right) \beta_{A} \Gamma_{\tau \tau}^{\tau(0)}-g\left(r_{c}\right) \beta_{A} \Gamma_{\tau \tau}^{r(0)}+\delta_{A B}\left(\Gamma_{\tau \tau}^{B(1)}+2 \Gamma_{\tau D}^{B(0)} v^{D}\right)=0 \\
\mathcal{O}\left(\epsilon^{2}\right):-f\left(r_{c}\right) \beta_{A}\left(\Gamma_{\tau \tau}^{\tau(1)}+2 \Gamma_{\tau D}^{\tau(0)} v^{D}\right)-g\left(r_{c}\right) \beta_{A}\left(\Gamma_{\tau \tau}^{r(1)}+2 \Gamma_{\tau D}^{r(0)} v^{D}\right) \\
+\delta_{A B}\left(\Gamma_{\tau \tau}^{B(2)}+2 \Gamma_{\tau D}^{B(1)} v^{D}+\Gamma_{C D}^{B(0)} v^{C} v^{D}\right)=0 \\
\mathcal{O}\left(\epsilon^{3}\right):-f\left(r_{c}\right) \beta_{A}\left(\Gamma_{\tau \tau}^{\tau(2)}+2 \Gamma_{\tau D}^{\tau(1)} v^{D}+\Gamma_{C D}^{\tau(0)} v^{C} v^{D}\right)-g\left(r_{c}\right) \beta_{A}\left(\Gamma_{\tau \tau}^{r(2)}+2 \Gamma_{\tau D}^{r(1)} v^{D}+\Gamma_{C D}^{r(0)} v^{C} v^{D}\right) \\
+\delta_{A B}\left(\partial_{\tau} v^{B}+v^{C} \partial_{C} v^{B}+\Gamma_{\tau \tau}^{B(3)}+2 \Gamma_{\tau D}^{B(2)} v^{D}+\Gamma_{C D}^{B(1)} v^{C} v^{D}\right)=\partial_{\tau} v_{A}+v^{C} \partial_{C} v_{A} \\
+\frac{r_{c}}{2}\left(\frac{a_{1}}{r_{c}} \partial_{A} P_{\mathrm{eff}}+a_{2} \partial^{2} v_{A}-\frac{2}{r_{c}} f\left(r_{c}\right) v^{D} \partial_{D} \beta_{A}\right) \\
-f\left(r_{c}\right) \partial_{\tau} \beta_{A}+f\left(r_{c}\right)\left(\partial_{A} \beta_{D}-\partial_{D} \beta_{A}\right) v^{D} .
\end{gathered}
$$




\section{APPENDIX E: CALCULATION OF THE EXPANSION SCALAR $\Theta(4.26)$ UP TO $\mathcal{O}\left(\epsilon^{3}\right)$ ORDER FOR CHOICE I}

The expansion scalar as derived on the cutoff hypersurface $r=r_{c}$ has the following form:

$$
\begin{aligned}
\Theta= & \underbrace{-\gamma^{\tau \tau}\left(\Gamma_{\tau \tau}^{\tau} v_{\tau}+\Gamma_{\tau \tau}^{r} v_{r}+\Gamma_{\tau \tau}^{A} v_{A}\right)}_{\text {expression 1 }}+\underbrace{-2 \gamma^{\tau A}\left(\Gamma_{\tau A}^{\tau} v_{\tau}+\Gamma_{\tau A}^{r} v_{r}+\Gamma_{\tau A}^{A} v_{A}\right)}_{\text {expression 2 }} \\
& +\underbrace{\gamma^{A B}\left(\partial_{A} v_{B}-\Gamma_{A B}^{\tau} v_{\tau}-\Gamma_{A B}^{r} v_{r}+\Gamma_{A B}^{D} v_{D}\right)}_{\text {expression } 3} .
\end{aligned}
$$

Evaluating "expression 1" from Eq. (E1) with the relevant $\Gamma$ 's obtained in Appendix A, we find its leading order to be $\mathcal{O}\left(\epsilon^{4}\right)$. Similarly, the leading-order contribution in "expression 2 " occurs at $\mathcal{O}\left(\epsilon^{4}\right)$. The leading-order behavior of "expression 3" occurs at $\mathcal{O}\left(\epsilon^{2}\right)$, and it is equivalent to $\partial_{A} v^{A}$ and vanishes at $\mathcal{O}\left(\epsilon^{3}\right)$.

\section{APPENDIX F: CALCULATION OF THE EXPANSION SCALAR $\Theta(5.16)$ UP TO $\mathcal{O}\left(\epsilon^{3}\right)$ ORDER FOR CHOICE II}

The "expansion parameter" as derived on the cutoff hypersurface $r=r_{c}$ has the following form:

$$
\begin{aligned}
\Theta= & \underbrace{\frac{1}{r_{c}}\left(\Gamma_{\tau \tau}^{\tau} v_{\tau}+\Gamma_{\tau \tau}^{r} v_{r}+\Gamma_{\tau \tau}^{A} \tilde{v}_{A}\right)}_{\text {expression } 1}+\underbrace{\left(2 \frac{f\left(r_{c}\right)}{r_{c}} \delta^{A B} \beta_{B}\right)\left(\Gamma_{\tau A}^{\tau} v_{\tau}+\Gamma_{\tau A}^{r} v_{r}+\Gamma_{\tau A}^{A} \tilde{v}_{A}\right)}_{\text {expression } 2} \\
& +\underbrace{\delta^{A B}\left(\partial_{A} \tilde{v}_{B}-\Gamma_{A B}^{\tau} v_{\tau}-\Gamma_{A B}^{r} v_{r}+\Gamma_{A B}^{D} \tilde{v}_{D}\right)}_{\text {expression } 3} .
\end{aligned}
$$

Evaluating "expression 1" from Eq. (F1) with the relevant $\Gamma$ 's obtained in Appendix A, we find its leading order to be $\mathcal{O}\left(\epsilon^{4}\right)$. Similarly, the leading-order contribution in "expression 2 " occurs at $\mathcal{O}\left(\epsilon^{4}\right)$. The leading-order behavior of "expression 3" occurs at $\mathcal{O}\left(\epsilon^{2}\right)$, and it is equivalent to $\partial_{A} v^{A}$ and vanishes at $\mathcal{O}\left(\epsilon^{3}\right)$.

[1] L. D. Landau and E. M. Lifshitz, Fluid mechanics, Course of Theoretical Physics (Pergamon Press, Oxford U.K., 1959).

[2] B. Lautrup, Physics of Continuous Matter, 2nd ed. (CRC Press, Hoboken, 2011).

[3] V.E. Hubeny, The fluid/gravity correspondence: A new perspective on the Membrane Paradigm, Classical Quantum Gravity 28, 114007 (2011).

[4] M. Rangamani, Gravity and hydrodynamics: Lectures on the fluid-gravity correspondence, Classical Quantum Gravity 26, 224003 (2009).

[5] T. Damour, Quelques Proprietes Mecaniques, Electromagnetiques, Thermodynamiques et Quantiques des trous noir (Thèse de doctorat d'Etat, Université Paris 6, Paris, France, 1979).

[6] T. Padmanabhan, Entropy density of spacetime and the Navier-Stokes fluid dynamics of null surfaces, Phys. Rev. D 83, 044048 (2011).

[7] E. Gourgoulhon and J. L. Jaramillo, A $3+1$ perspective on null hypersurfaces and isolated horizons, Phys. Rep. 423, 159 (2006).
[8] K. Bhattacharya, B. R. Majhi, and D. Singleton, Fluidgravity correspondence in the scalar-tensor theory of gravity: (in)equivalence of Einstein and Jordan frames, J. High Energy Phys. 07 (2020) 018.

[9] S. Kolekar and T. Padmanabhan, Action principle for the fluid-gravity correspondence and emergent gravity, Phys. Rev. D 85, 024004 (2012).

[10] R. H. Price and K. S. Thorne, Membrane viewpoint on black holes: Properties and evolution of the stretched horizon, Phys. Rev. D 33, 915 (1986).

[11] C. Eling, I. Fouxon, and Y. Oz, The incompressible NavierStokes equations from membrane dynamics, Phys. Lett. B 680, 496 (2009).

[12] E. Gourgoulhon and J. L. Jaramillo, New theoretical approaches to black holes, New Astron. Rev. 51, 791 (2008).

[13] S. Bhattacharyya, S. Minwalla, and S. R. Wadia, the incompressible non-relativistic Navier-Stokes equation from gravity, J. High Energy Phys. 08 (2009) 059.

[14] S. Bhattacharyya, V. E. Hubeny, S. Minwalla, and M. Rangamani, Nonlinear fluid dynamics from gravity, J. High Energy Phys. 02 (2008) 045. 
[15] S. Bhattacharyya, R. Loganayagam, S. Minwalla, S. Nampuri, S. P. Trivedi, and S. R. Wadia, Forced fluid dynamics from gravity, J. High Energy Phys. 02 (2009) 018.

[16] G. Policastro, D. T. Son, and A. O. Starinets, From AdS/CFT correspondence to hydrodynamics, J. High Energy Phys. 09 (2002) 043.

[17] I. Bredberg, C. Keeler, V. Lysov, and A. Strominger, From Navier-Stokes to Einstein, J. High Energy Phys. 07 (2012) 146.

[18] G. Compere, P. McFadden, K. Skenderis, and M. Taylor, The Holographic fluid dual to vacuum Einstein gravity, J. High Energy Phys. 07 (2011) 050.

[19] D. Brattan, J. Camps, R. Loganayagam, and M. Rangamani, CFT dual of the AdS Dirichlet problem: Fluid/Gravity on cut-off surfaces, J. High Energy Phys. 12 (2011) 090.

[20] N. Pinzani-Fokeeva and M. Taylor, Towards a general fluid/ gravity correspondence, Phys. Rev. D 91, 044001 (2015).

[21] T. Z. Huang, Y. Ling, W. J. Pan, Y. Tian, and X. N. Wu, From Petrov-Einstein to Navier-Stokes in spatially curved spacetime, J. High Energy Phys. 10 (2011) 079.

[22] S. De and B. R. Majhi, Fluid description of gravity on a timelike cut-off surface: Beyond Navier-Stokes equation, J. High Energy Phys. 01 (2019) 044.

[23] C. Y. Zhang, Y. Ling, C. Niu, Y. Tian, and X. N. Wu, Magnetohydrodynamics from gravity, Phys. Rev. D 86, 084043 (2012).

[24] G. Chirco, C. Eling, and S. Liberati, Higher curvature gravity and the holographic fluid dual to flat spacetime, J. High Energy Phys. 08 (2011) 009.

[25] X. Bai, Y. P. Hu, B. H. Lee, and Y. L. Zhang, Holographic charged fluid with anomalous current at finite cutoff surface in Einstein-Maxwell gravity, J. High Energy Phys. 11 (2012) 054.

[26] R. G. Cai, T. J. Li, Y. H. Qi, and Y. L. Zhang, Incompressible Navier-Stokes equations from Einstein gravity with ChernSimons term, Phys. Rev. D 86, 086008 (2012).

[27] D. C. Zou, S. J. Zhang, and B. Wang, Holographic charged fluid dual to third order Lovelock gravity, Phys. Rev. D 87, 084032 (2013).

[28] Y. P. Hu, Y. Tian, and X. N. Wu, Bulk viscosity of dual fluid at finite cutoff surface via gravity/fluid correspondence in Einstein-Maxwell gravity, Phys. Lett. B 732, 298 (2014).
[29] R. G. Cai, L. Li, and Y. L. Zhang, Non-Relativistic fluid dual to asymptotically AdS gravity at finite cutoff surface, J. High Energy Phys. 07 (2011) 027.

[30] T. Z. Huang, Y. Ling, W. J. Pan, Y. Tian, and X. N. Wu, Fluid/gravity duality with Petrov-like boundary condition in a spacetime with a cosmological constant, Phys. Rev. D 85, 123531 (2012).

[31] D. Anninos, T. Anous, I. Bredberg, and G. S. Ng, Incompressible fluids of the de Sitter horizon and beyond, J. High Energy Phys. 05 (2012) 107.

[32] Y. Ling, C. Niu, Y. Tian, X. N. Wu, and W. Zhang, Note on the Petrov-like boundary condition at finite cutoff surface in gravity/fluid duality, Phys. Rev. D 90, 043525 (2014).

[33] C. Eling, A. Meyer, and Y. Oz, The relativistic rindler hydrodynamics, J. High Energy Phys. 05 (2012) 116.

[34] J. Berkeley and D. S. Berman, The Navier-Stokes equation and solution generating symmetries from holography, J. High Energy Phys. 04 (2013) 092.

[35] V. Lysov, Dual fluid for the kerr black hole, J. High Energy Phys. 06 (2018) 080.

[36] X. Wu, Y. Ling, Y. Tian, and C. Zhang, Fluid/gravity correspondence for general non-rotating black holes, Classical Quantum Gravity 30, 145012 (2013).

[37] C. J. Chou, X. Wu, Y. Yang, and P. H. Yuan, Rotating black holes and Coriolis effect, Phys. Lett. B 761, 131 (2016).

[38] T. Padmanabhan, Thermodynamical aspects of gravity: New insights, Rep. Prog. Phys. 73, 046901 (2010).

[39] S. De, S. Dey, and B. R. Majhi, Effective metric in fluidgravity duality through parallel transport: A proposal, Phys. Rev. D 99, 124024 (2019).

[40] T. Padmanabhan, Gravitation: Foundations and Frontiers (Cambridge University Press, Cambridge, England, 2010).

[41] K. Parattu, B. R. Majhi, and T. Padmanabhan, Structure of the gravitational action and its relation with horizon thermodynamics and emergent gravity paradigm, Phys. Rev. D 87, 124011 (2013). 